\title{
Attività sismica e neotettonica della Valle del Belice
}

\author{
(Seismic activity and neotertonic of the Belice Valley. Sirily)
}

A. BOTTuRI (*)

Ricevito il 18 Ottobre 1972

\begin{abstract}
Russuxto. - Con rignado a precedenti ricerehe condotte da vari Autori, sono esposti i risultati di uno studio su 14 terremoti con $M \geqslant 4,4$ (Messina Lniv.) della sequenza sismica che nel 1968 ha interessato la Sicilia centro-oceddentale ed in modo particolare la Valle del Belice.

I iantore, utilizzando i tempi inizio delle $P(0 \quad P n)$ in wn sulficientc numero di stazioni ancle Iontane, detcrmina i parametri ipocentrali avvalendosi dol metodo statistico di Caloi opportunamente tradotto in Fortran IV per consentire l'impiego di nn claboratore elettronico IJ3 स्स 11-30.

Lanalisi dei dati ottenuti, in aceordo con l'ipotesi di correlazione sismicitì-neotettonica avanzala da alcuni Autori, nel qualro delle conoscenze geo-unorfologiche della zona sinistrata, i a favore di una rostanziale superficialitio degli ipocentri $(h \leqslant 28 \mathrm{~km})$.

Una snecessiva determinazione, partendo da quest'ultiua assunzione, conferma la non casualitì della precedente distribuzione epientrale.

Infinc, lo stutio dei versi dei primi impulsi generati dalla scossa prineipale $(X Y=5,9)$ porge direttamente un altro dato a sostegno rel nesso sismicitimeotettonica ed indirettamente un ulteriore elemento a favore della sujerficialità degli iprocentri.
\end{abstract}

Sumans, - The results on the study of 14 earthiuakes having II $\geqslant 4,4$ (Messina Iniv.) and ocemred in 1968 in the Western-Center of Sicily, with particular evidence in the Belice Valley, are reported with regard to preseding studies mate by few rescarchers.

(*) Istituto Geofisien e Geodetico, Linirersitì degli Studi, \$Lessina. 
The author, using the $P(o r P$ r arrival times in an adequate whole of stations, determines the hypocentral parameters by Caloi statistical method previously translated into fortran ]V in order to employ an IIBM $11-30$ electronic computer.

The obteined data analisis in accordance with a seismicity-neotectonic correlation hypolhesis, supported by some author in geo-morphological ont. line of damaged zone, shows sulstantial shallowness for the hypoesnters $(h \leqslant 28 \mathrm{~km})$.

A furtlue determination, starting fou the previous statement, intlicates uncasuality of preceding cpicentral arrangement.

ln the end, studyng the first-motion directions produced to main shock $(1 /-5,9)$ another result is founded favomable to seismicity-neo. tectonic cmploy and indirectly also a furthor element bringing evislence in support of hypocentral shillowness.

1. - Un discreto numero di studiosi ha pubblicato i risulati di varie ricercle conclotte intorno all'intenso fenomeno sismico che, per grin parte rel 1968, ha interessato tutta la Sicilia centro occirlentale ed in moslo particolare la Valle del Belice e la parte orientale della Valle di Mazara. Gli studi sono stati condotti muovendo da conoscenze generalmente diverse, adottando metoriche diflerenti, ron prospetive diverse anche se spesso complementadi.

Bosi, Cavallo e Manfrerlini $\left({ }^{2}\right)$ ad esempio, delineate le caratteristiche greomorfologiche dellarea serle degli eventi sismici, operano in via preliminare alla individuazione di elementi atbi a sostenere l'ipotesi, avanzala precerlentemente da Manfrerlini, the l'atlivila sismica sviluppatasi, più che alla presenza di faglie attive, possa essere messa in relazione con l'esistenza di zone di giunzione fra aree a dilterente evoluzione geologica. Tali giunzioni potrebbero essere individuate, con una certa approssimazione, dagli allineamenti Montevago-BisaquinoCorleone a Castelvetrano-MI,te Finestrelle (fig. 1). La ricerca i pero solamente delineata, mancando agli futori l'apporto dei dabi derivanti dal rilevamento sismico strumentale.

Con riferimento alle caratteristiche geologico-tecniche delle formazioni di fondazione dei aentri abitati, noncho alle caratteristiche feniche rlelle costruzioni, è presentato poi nu quadro degli efletti macrosismiri sui terreni e sui manufati delle località maggiormente colpite.

Successivanente, Joe Panfilis e Marcelli (5) espongono i risultati delle osservazioni eftettuate per i terremoli piit forti e presentano un 


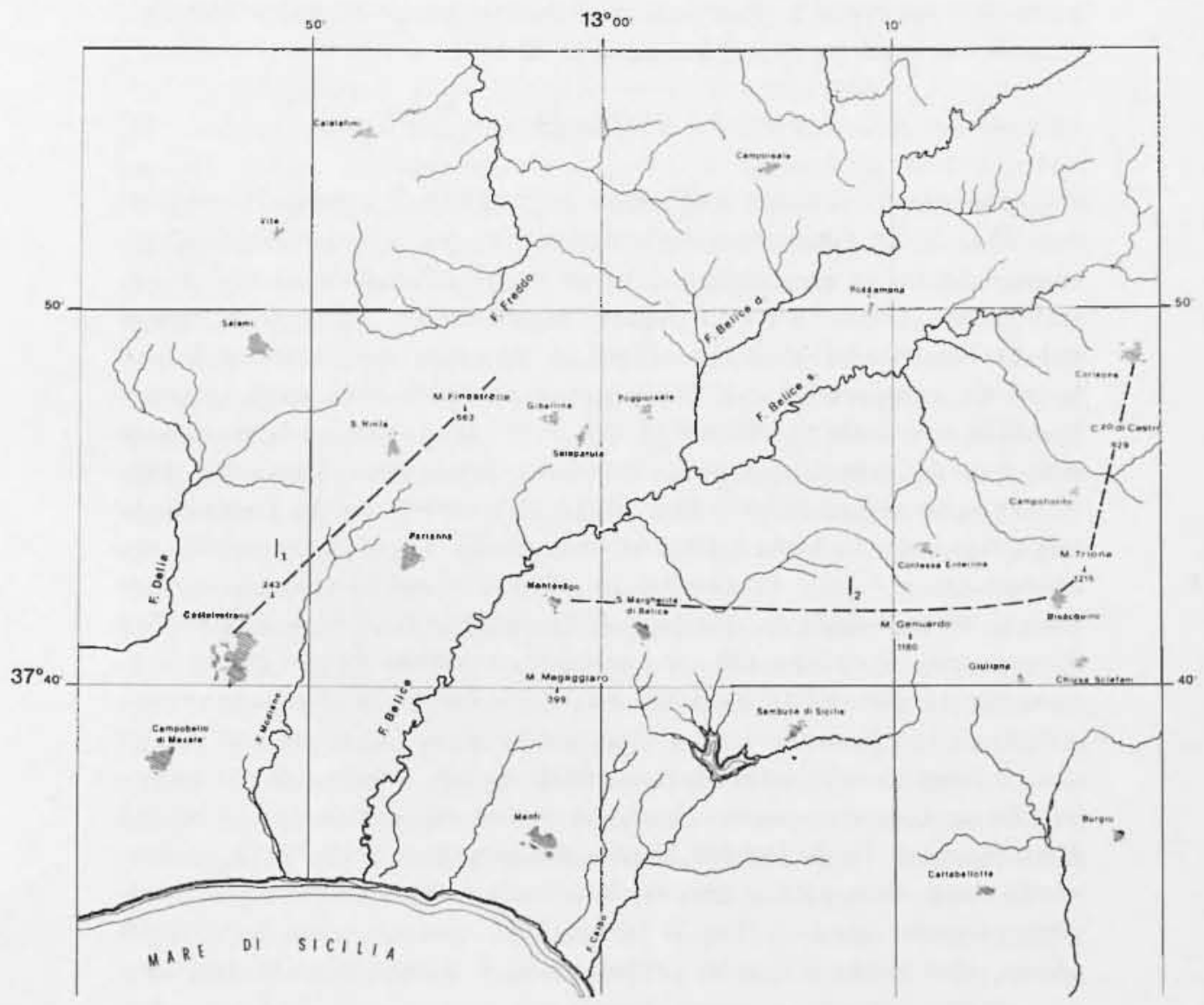

Fig. 1

diagramma della frequenza rei sismi fino al 10 (rimgno 1968. Notevole rilievo d̀ dato alla cauterizzazione masosisnica reggli eventi che dे riassunta, per quanto concerne gli effetti crlobali, neila carta rlelle isohlabe. Segrue nua rassegna dellativitio sismio della Sirilia centrooccidentale con jarticolare riferimento alla zona del Belice ed al Palermitano.

Girlancia ( $\left.{ }^{8}\right)$, ne] Ginguo dello stesso anno, pubblisa lo sturlio rlei 154 terremoti registrati a Messina Univ. ral 14 Gennaio al $1^{\circ}$ Giugno 1968 erl axenti origine nella Sicilia occidentale. E determinata la ma- 


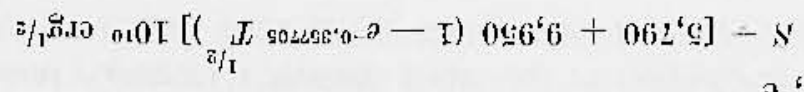

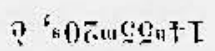

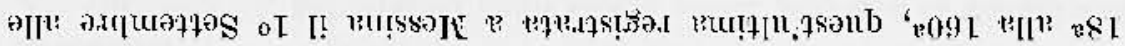

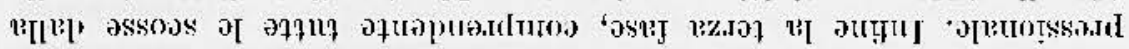

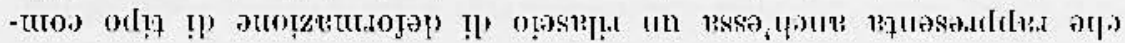

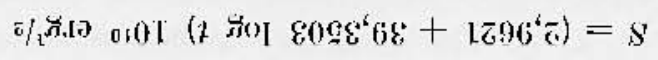

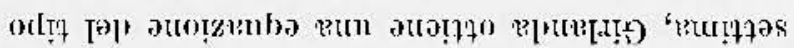

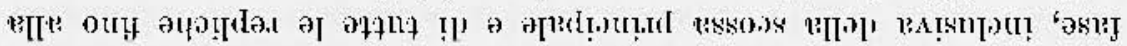

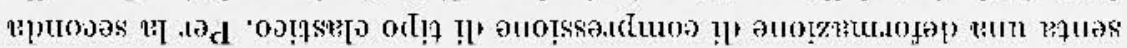

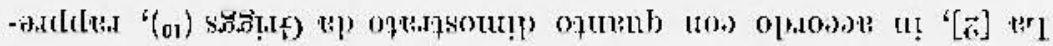

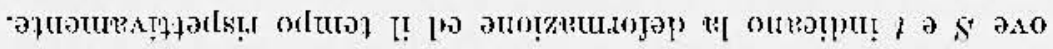
$[\pi]$

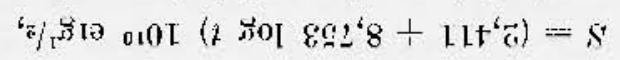

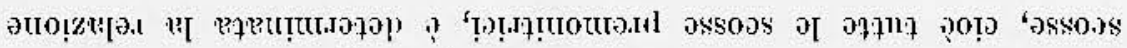

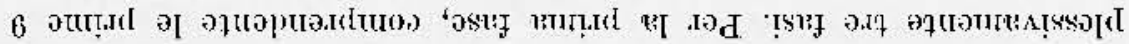

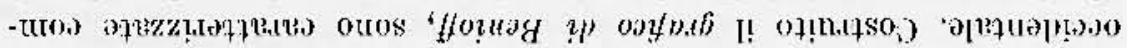

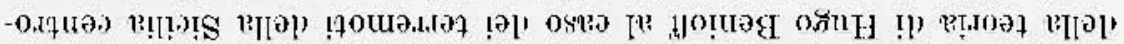

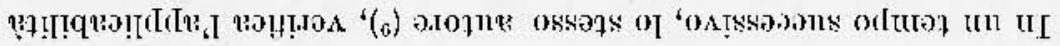

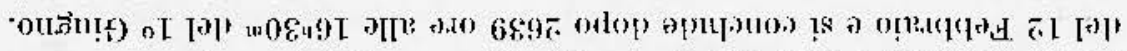

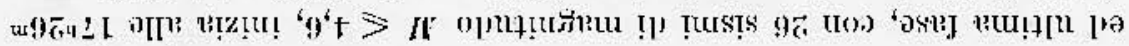

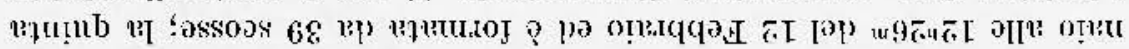

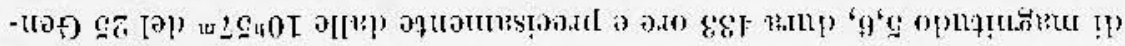

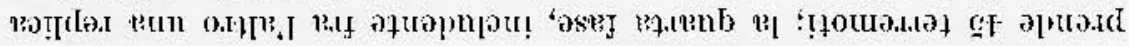

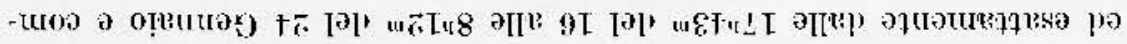
ә.10 द.8

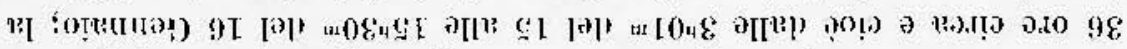

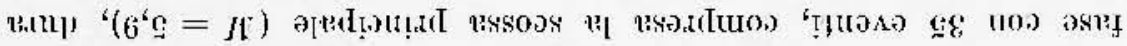

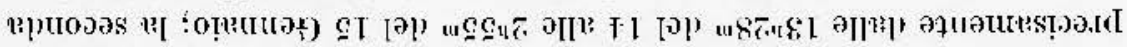

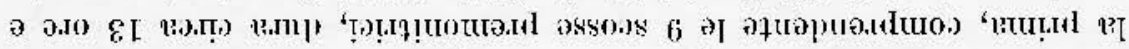

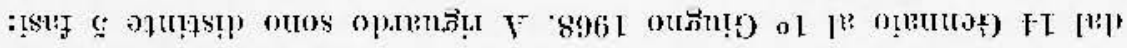

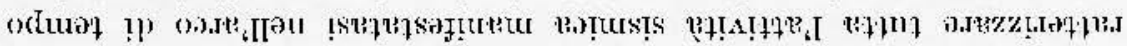

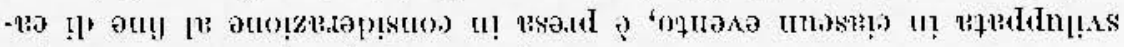

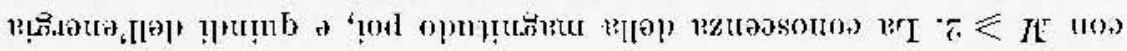

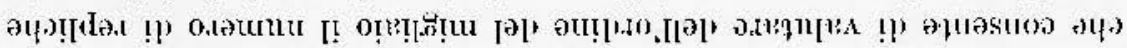

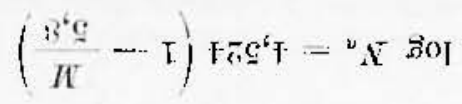

$\varepsilon<\pi$ op

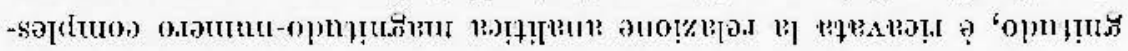


ove $T=t-1,18$ (essento 1,18 il tempo della $17^{\mathrm{a}}$ scossal contato a partire dalle $00^{ }$del 11 Gennaio). La [1] è del tipo previsto da Michelson ( $\left.{ }^{13}\right)$ nel processo (li recupero di erepp distorsionale.

J'autore conclusle allermando che le due fasi clistinte carniterizzate, quella di compressione e guclla di flistorsione, ben si arcorderebbero con il comportamento tipico di un'muica faglia gioentes secondo il meccusismo proposto dalla teoria di Beniofl, che pertanto potrebbe essere assunto per una schematizzazione flella sequenza sismica siciliana.

Valle (17), lammo scguente, ripropone la teoria dello elustic robound e determina per ciascun evento la percentuale di energia $\theta$ di deformazione liberate per onde clastiche. Calcola il rendimento e la variazione di rendimento previsto ex osservato nel passaggio da una replica alla successiva e pone in particolare rilievo la valutazione dellandamento della seric delle repliche al fine di operare un tentativo di previsione circa l'evolversi flel fenomeno sismico.

Marecli e Iamnochia (12), infine, pubblicano i risultati di una rleterminazione analitiol delle coor catesi nei primi tre giorni di atività. Il risultato fli maggior rilievo è quello relativo alla profontiti rlegli ipocentri calcolati. I due autori infati, in rlisaccorio con quanto ritenuto o sostennto da tutti i ricereatori fin qui citati, che si sono dichiamati a favore della superficialiti o comunque modestissima profondita degli ipocentri, ubicano 9 dei 10 fuochi al rlisotto rlel limite inferiore rlella crosta terrestre.

Più precisamente, se si escluce la seconda scossa premonitrice $\left(I I=13^{\mathrm{n}} 15^{\mathrm{m}} \cdot 16 \mathrm{~s}, 73\right)$ per cui è valutata la profondità $h=28,1 \mathrm{~km}$, tutte le altre, compresa la scossa piu forte, avrebbero origrine fra $h=34$ e $h=5 \pi \mathrm{km}$ (Tabella I).

Concludendo, i terremoti rella Sieilia centro-occidentale sono stati oggetto ali indagine per vari ricercatori i quali hanno sffrontato lo sturlio rlel fenomeno con metoriche e prospettive alferenti. I risultati ottenuti, anche in consirlerazione flel fatio che la zona sede dell attivitit sismica in questione era fino al 13 Gennaio 1968 consirlerata asismica, devono ritenersi di indubbio interesse.

Premesso eio, non avvertiremmo alcuma neressità nel partecipare alle ricerele sui termoti del Belice, pure se con un contributo morlesto, se non rilevassimo, da un lato la presenza di alcune incongruenze, e dallaltro l'utilitia di approfondire alcuni aspetti del complesso fenomeno anche al fine di verificare la legittimita di talune ipotesi. 


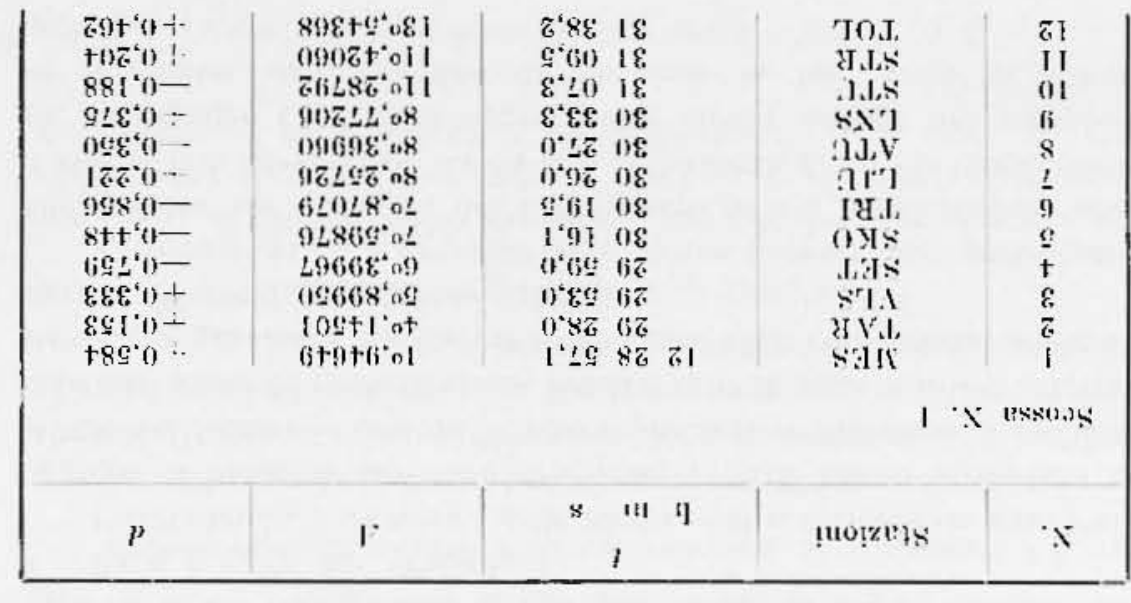

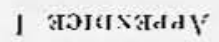

\begin{tabular}{|c|c|c|c|}
\hline 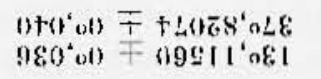 & 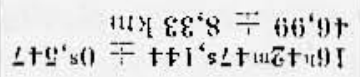 & $8965 \mathrm{~T} \cdot \mid-9)$ & 0) 1 \\
\hline 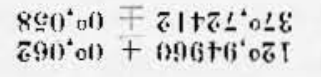 & 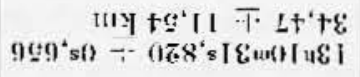 & $896 \mathrm{~T}-\mathrm{I}-9]$ & $\mathbf{b}^{2}$ \\
\hline 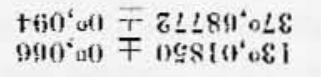 & 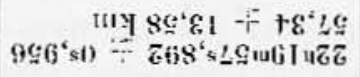 & $896) \mathrm{I}-\mathrm{I}-9 \mathrm{I}$ & 8 \\
\hline 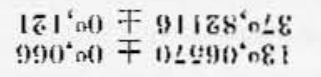 & 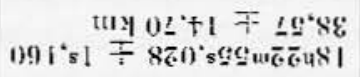 & $8961-1-91$ & 1 \\
\hline 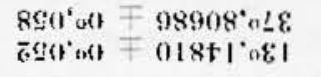 & 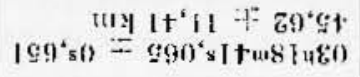 & $8961-1-61$ & 9 \\
\hline 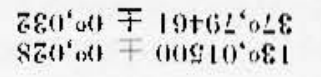 & 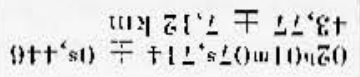 & $8961-1-1-1$ & 9 \\
\hline 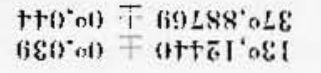 & 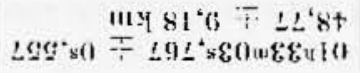 & $8961-7-61$ & $\hbar$ \\
\hline 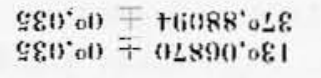 & 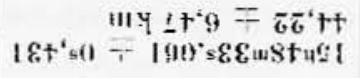 & $89611-1-+1$ & $\varepsilon$ \\
\hline 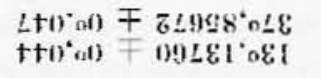 & 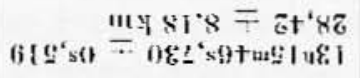 & $80161-1 \cdot+1$ & 7 \\
\hline 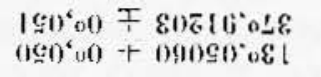 & 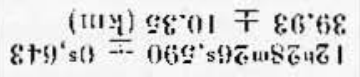 & $89161-1 \cdot+1$ & I \\
\hline 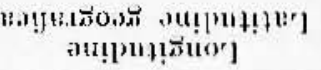 & 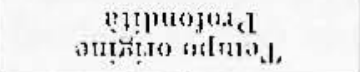 & :?) & $\therefore$ \\
\hline
\end{tabular}

I $)[]^{2}$ 


\begin{tabular}{|c|c|c|c|c|c|}
\hline e(og' $\{1\}+$ & $(5++0) L \circ \% \&$ & L'EI & $\mathrm{gg}$ & †) & 81 \\
\hline gog' 0 & t86896to & $0^{2} \mathrm{IS}$ & 89 & 'Tild & $2 \mathrm{I}$ \\
\hline $82600-$ & $8 \&(1 \leq * * \circ) 1$ & 0 & 69 & IVY.I. & $9 \mathrm{I}$ \\
\hline $3+20 \cdots$ & Eв\&z: ०६1 & $t^{*} \varepsilon t$ & 19 & IO.L. & gI \\
\hline $081^{\circ} 0--$ & lotot'oll & $0)^{4} 91$ & IG & प.L. & +1 \\
\hline $8 \pi 20 \div$ & 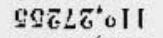 & $8^{6}+1$ & Is & A.LS & $\$ 1$ \\
\hline+90()$^{4}(0 ;-$ & 8001 L'mol & $\mathrm{e}^{\circ} 90$ & Is & ₹४Я & 61 \\
\hline$+49^{*}(0)$ & 2880901 & 0 & 19 & III. & II \\
\hline$+10 \%-$ & $86891^{\prime}$ orli & $0)^{6} 60$ & (1) & .101 & $0 \mathrm{I}$ \\
\hline 8L8'1) - & $88 \angle 6 I^{\circ} \mathrm{ob}$ & $069+$ & $0 \mathrm{~g}$ & 'IN⿴囗十 & 6 \\
\hline gige - & 108g:08 & 1) $60 t$ & $0 \mathrm{G}$ & $\operatorname{SiN}^{-1} \mathrm{I}$ & 8 \\
\hline $9+20$ & $208+6 \cdot 08$ & $0+78$ & $0 \underline{G}$ & A $\mathrm{P}^{\prime \prime I}$ & 2 \\
\hline $9800^{4} 13-$ & (1)+139)8०01 & $13^{*} \angle z$ & $0 \leqq$ & $14 . \mathrm{L}$ & 9 \\
\hline $888^{\prime} 0-$ & $0 L(H 3))^{6} \circ L$ & I'\&6 & $0 g$ & (1) Y5 & g \\
\hline $2+\sigma^{\circ} 0$ & $299166^{4} \mathrm{~g}$ & (1) 600 & $0 \mathrm{~g}$ & $\leqslant[1.1$ & + \\
\hline $88 \sigma^{\circ} 1$ & $8996 t^{\prime}$ of & 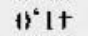 & $6 t$ & $.10 \mathrm{~V}$ & $\varepsilon$ \\
\hline (30)8 0 - & seg9o0 ot & $8^{\prime} z \varepsilon$ & $6 t$ & itot & 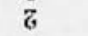 \\
\hline \multirow[t]{2}{*}{$\left.815^{\circ} 0\right)^{--}$} & $16696 \div 1$ & $\sigma^{\circ} 80$ & 6t 91 & S. & 1 \\
\hline & & & & \multicolumn{2}{|c|}{$\varepsilon \cdot \mathrm{N}$ 1tssons } \\
\hline $96 z^{\circ} 0-$ & $\varepsilon+10+\circ 98$ & +46 & $8 z$ & oाrg & $\varepsilon \bar{c}$ \\
\hline $6810-$ & $2800 \sigma^{\circ} 088$ & $13 \div 11$ & $8 \overline{6}$ & \%()\& & $\overline{6} 6$ \\
\hline $8 \pm 6 \% 0+$ & $\varepsilon \sigma \leq+0^{\circ} \mathrm{a} !$ & $13^{*} 901$ & $9 \overrightarrow{6}$ & YIIL & 16 \\
\hline LI10 $0+$ & $2 \& 16 \varepsilon^{6} \circ 6^{\circ}$ & $0^{*} 8 t$ & 96 & Т.1ย & $0 z$ \\
\hline $9860+$ & $+288 t^{\circ} \circ \mathrm{QSE}$ & $\mathrm{g}^{*} \mathrm{It}$ & 66 & $\mathrm{OH}^{\prime} \mathrm{I}$ & bi \\
\hline $86+0^{\circ} 0-$ & 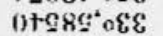 & $\therefore 97$ & 63 & DX์ & 81 \\
\hline $8620-$ & $\varepsilon 1+8+\circ 0 \varepsilon$ & $0^{2}+2$ & 16 & U1। & 21 \\
\hline I\&\&'I- & $9+s 9+096$ & 968 & $1 z$ & 政行 & 9 [ \\
\hline $6 \mathrm{JH}^{4} \mathrm{I}+$ & $\csc +1$ ost & $00 z$ & I $z$ & ЯIV.L & GI \\
\hline$\left.+88^{4} 1\right)+$ & casitotz & $\varepsilon^{\prime} \& 11$ & 16 & solt & $t I$ \\
\hline$\angle 200$ & $826+6 \circ 86$ & 6228 & $0 \overline{6}$ & ห.רN & $\varepsilon 1$ \\
\hline $689^{6} 13+$ & †8968*0II & $6^{2} 6 \sigma$ & $8 \mathrm{I}$ & Ону & 71 \\
\hline $099418-$ & 98688011 & +66 & 81 & nLS & II \\
\hline $8+00^{\circ}(1 \div$ & $68998^{\circ} 08$ & $1)^{6}+\mathrm{t}$ & 21 & $\therefore N^{\prime} 1$ & 01 \\
\hline $181^{\circ} 0-$ & $2160+0^{\circ} 08$ & $g^{*} \geq t$ & 21 & A.LY & 6 \\
\hline $6 z \sigma^{\circ}(1)$ & $9 \angle 698 \div 08$ & $0^{\circ} \iota t$ & 21 & GI'T & 8 \\
\hline $80 \pi \cdot 1 \div$ & $1+880601$ & 1) 8 เ & 21 & I $4 . \mathrm{L}$ & 1 \\
\hline $\left.6(6+)^{\circ}\right)-$ & 1238901 & I"थ\& & 21 & 0уง & 4 \\
\hline $96+50-$ & 80\&ce"n! & g'8I & 21 & LCOLS & $\$$ \\
\hline $698^{\circ} 0-$ & $9 b+860 \mathrm{~s}$ & $8^{6} 61$ & 41 & SrIA & $t$ \\
\hline $119^{\circ} 0-$ & $0 \angle 8$ [9. of & $1)^{\circ} \mathrm{Gg}$ & !ा & $.10 \mathrm{~V}$ & 8 \\
\hline $698^{\circ}(0)$ & $0128 \mathrm{I}^{\circ} \mathrm{t}$ & $q^{\prime} \iota t$ & 91 & ITO\& & $\tilde{6}$ \\
\hline \multirow[t]{2}{*}{$16+0-$} & $+1+66 \circ \circ$ & 299 & 9181 & STIT & i \\
\hline & & & & \multicolumn{2}{|c|}{$z x$ ussous } \\
\hline $99 \sigma^{\prime} 0 \div$ & 96026 ogs & E'ç & $0 t$ & OIC.II & $8 \mathrm{I}$ \\
\hline $918^{\circ} 0 \div$ & $96 t+9608$ & 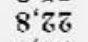 & 98 & ו'וקי & 21 \\
\hline $0 \cos { }^{\circ} 0-$ & 610169'०\&8 & 200 & $9 \varepsilon$ & !) & 91 \\
\hline $8 \overline{6} 0^{\circ} 0-$ & $0 c \overline{c t g}$ ofes & $0^{5} 6 \varepsilon$ & $t \%$ & (IC) & @i \\
\hline $6 \leq 00^{\circ} 0$ & $9696 \bar{\sigma} \circ 8 \overline{6}$ & $6)^{\prime} 61$ & $+\varepsilon$ & 1.10 & +1 \\
\hline $2 \leq+0+$ & $68 \pm 98 \circ 91$ & $0)^{*}=1$ & $6 \& \approx 1$ & IIV V.L & 81 \\
\hline$p$ & $\nabla$ & $s$ & II I & !tu!zu।s & N \\
\hline
\end{tabular}




\begin{tabular}{|c|c|c|c|c|c|}
\hline \multirow{2}{*}{$x}$. & \multirow{2}{*}{ Stazinni } & \multicolumn{2}{|r|}{$t$} & \multirow{2}{*}{.1} & \multirow[t]{2}{*}{$d$} \\
\hline & & h 111 & $\mathrm{~s}$ & & \\
\hline 19 & Q[i] & 155 & 645,3 & $44^{\circ} .75566$ & 0,139 \\
\hline 20 & $\mathrm{Bl}: \mathrm{I}$, & & 836,0 & $59^{\circ}, 506127$ & 0,506 \\
\hline 2) & BRIV & & (9) 48,3 & $70^{\circ}, 90197$ & $-0,720$ \\
\hline 22 & $130 \%$ & & 056,0 & $82^{\circ}, 87274$ & 0,143 \\
\hline 23 & J3LT & & (1) 57,2 & $83^{\circ}, 30446$ & $--0,879$ \\
\hline 24 & W:JIO & & 101,2 & $83^{\circ}, 94000$ & $-0,111$ \\
\hline 25 & TFO & & 38,6 & 910,56789 & $\div 0,574$ \\
\hline \multicolumn{6}{|c|}{ Scossa X. 4} \\
\hline 1 & MES & 013 & $3 \quad 34,4$ & 20.09213 & $-0,689$ \\
\hline 2 & RCI & & $3 \quad 35,7$ & $2^{0}, 14890$ & $-0,209$ \\
\hline 3 & ROO.J] & & 403,2 & $4^{\circ}, 06867$ & 0.078 \\
\hline 4 & SE'T' & & $4 \quad 33,5$ & $6^{\circ}, 25491$ & $-0,683$ \\
\hline 5 & XIION & & $4 \quad 47,2$ & $7^{\circ}, 21797$ & $-0,508$ \\
\hline 6 & ISO & & $+54,8$ & $7^{\circ}, 74226$ & 0,199 \\
\hline 7 & ALG & & 457,0 & $7^{\circ}, 95112$ & - 0,925 \\
\hline 8 & TRI & & 458,0 & 70.88330 & $-1,026$ \\
\hline 9 & 13120 & & $5 \quad 11,6$ & 80,96962 & $-0,491$ \\
\hline 10) & RSL & & 515,8 & $9^{\circ}, 14696$ & $1,1,263$ \\
\hline 11 & B RA & & 537,5 & $10^{\circ}, 75760$ & 0,802 \\
\hline 12 & STE & & 543,9 & $11^{0}, 25726$ & $\begin{array}{l}0,423 \\
-4\end{array}$ \\
\hline 13 & STR & & 545,5 & $11^{0}, 37779$ & 0,379 \\
\hline 14 & (ARE & & 551,5 & 110,91327 & $-0,891$ \\
\hline $1 \overline{5}$ & IsT & & $\begin{array}{ll}6 & 04,4\end{array}$ & $12^{\circ}, 80508$ & $\therefore 0,031$ \\
\hline 16 & ISK & & 605,1 & $12^{\circ}, 86401$ & $-0,054$ \\
\hline 17 & TA.II & & 651,0 & $16^{\circ}, 3018 \overline{5}$ & $+1,018$ \\
\hline 18 & HLW & & 702,0 & 170,21433 & $\div 0,489$ \\
\hline 19 & JER & & 726,0 & 190,24505 & $-0,313$ \\
\hline 20 & NER & & $8 \quad 12,9$ & $23^{\circ}, 86903$ & $-0,774$ \\
\hline 21 & $\mathrm{BNG}$ & & 943,8 & $33^{\circ}, 71278$ & $-1,039$ \\
\hline 22 & J.IC: & & 957,4 & $35^{\circ}, 46405$ & $-0,411$ \\
\hline 23 & QUE & & $1 \quad 15,2$ & $44^{\circ}, 88594$ & $-0,491$ \\
\hline 24 & liRW & & 417,6 & $70^{\circ}, 90850$ & $-0,911$ \\
\hline 25 & $\mathrm{~B} 0 \mathrm{O} \%$ & & $52 \overline{7}, 5$ & $83^{\circ}, 13091$ & +0.860 \\
\hline 26 & W 310 & & 530,3 & $83^{\circ}, 84948$ & $-0,005$ \\
\hline 27 & DOUG & & 549,2 & $87^{\circ}, 80173$ & $-0,568$ \\
\hline 28 & TFO & & 607,8 & $91^{\circ}, 48979$ & $+0,681$ \\
\hline \multicolumn{6}{|c|}{ Scossa N. 5} \\
\hline 1 & MES & 020 & 139,4 & $2^{\circ}, 00868$ & $\therefore 0,922$ \\
\hline 2 & ROMI & & $2 \quad 09,2$ & $4^{0}, 09322$ & 1,021 \\
\hline 3 & VLS & & 234,1 & $5^{\circ}, 96062$ & $-0,498$ \\
\hline 4 & SE'T & & 239,0 & 60,33732 & $-0,950$ \\
\hline 5 & MON & & 252,3 & $7^{\circ}, 28113$ & $-0,888$ \\
\hline 6 & SKO & & 257,8 & 70,65866 & $-0,669$ \\
\hline 7 & 150 & & 300,2 & 70,80490 & $-0,31 \overline{5}$ \\
\hline 8 & TRI & & 301,0 & 70,89400 & $-0,764$ \\
\hline 9 & ALG & & $3 \quad 04,0$ & $8^{\circ}, 05086$ & $+0,035$ \\
\hline 10 & $\Lambda \mathrm{TU}$ & & $3 \quad 08,4$ & $8^{\circ}, 43037$ & $-0,880$ \\
\hline 11 & INS & & $3 \quad 14,0$ & $8^{\circ}, 75947$ & $+0,166$ \\
\hline 12 & YOL & & 334,0 & $10^{\circ}, 17488$ & $\div 0,647$ \\
\hline
\end{tabular}




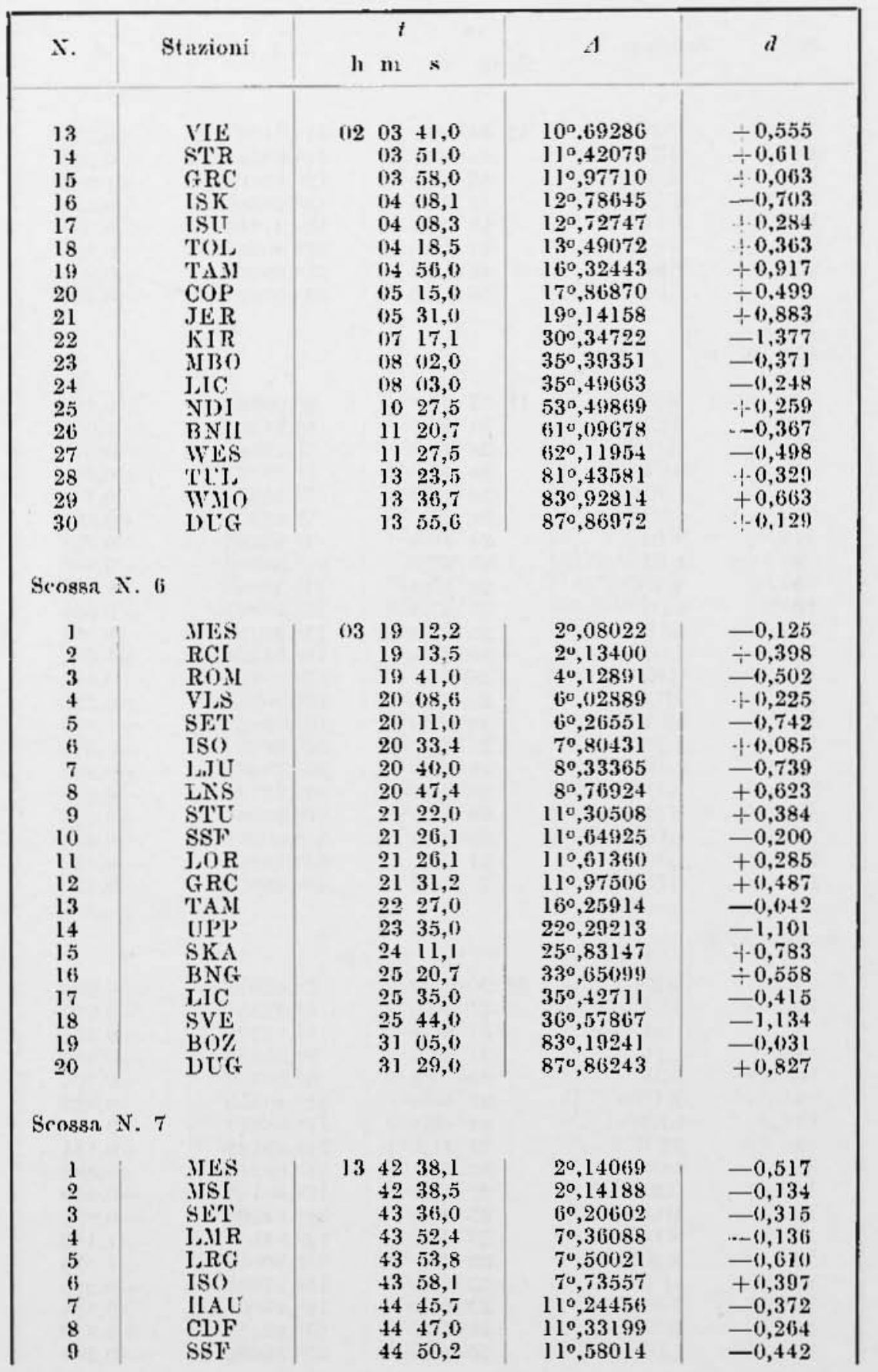




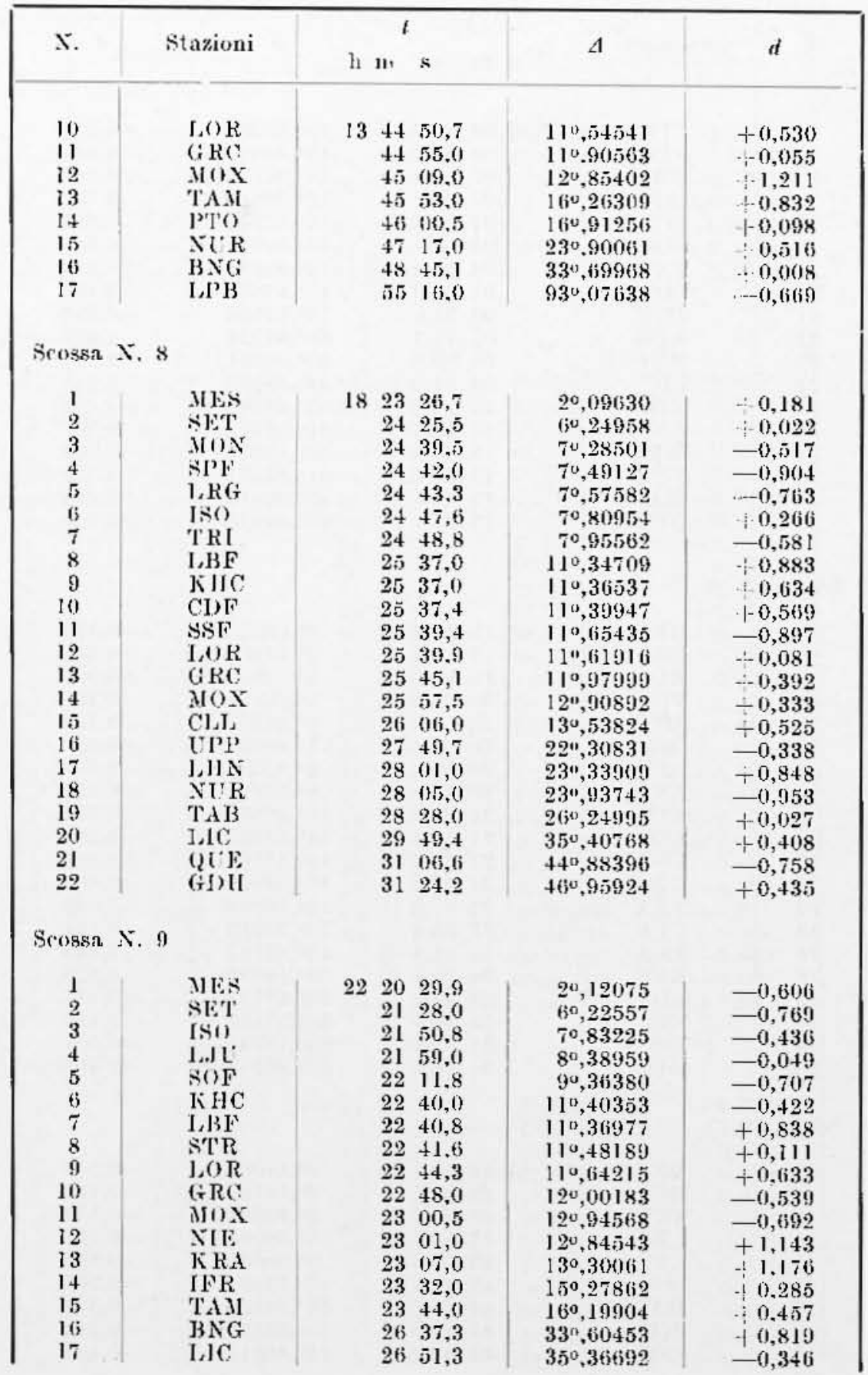




\begin{tabular}{|c|c|c|c|c|c|c|}
\hline K. & Stazioni & li & $\mathrm{m}^{t}$ & s & $\Delta$ & d \\
\hline $\begin{array}{l}18 \\
19 \\
20 \\
21 \\
22\end{array}$ & 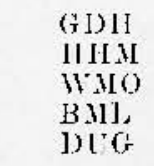 & 22 & $\begin{array}{l}28 \\
32 \\
32 \\
32 \\
32\end{array}$ & $\begin{array}{l}26,2 \\
15,6 \\
25,5 \\
37,1 \\
44,9\end{array}$ & $\begin{array}{l}46^{\circ}, 98572 \\
82^{\circ} .18745 \\
83^{\circ}, 91590 \\
86^{\circ}, 33397 \\
87^{\circ}, 88514\end{array}$ & $\begin{array}{r}-0,727 \\
-0,701 \\
+0,303 \\
-0,099 \\
-0,201\end{array}$ \\
\hline \multicolumn{7}{|c|}{ Scussa N. 10} \\
\hline $\begin{array}{r}1 \\
2 \\
3 \\
4 \\
5 \\
6 \\
7 \\
8 \\
9 \\
10 \\
11 \\
12 \\
13 \\
14 \\
15 \\
16 \\
17 \\
18 \\
19 \\
20 \\
21 \\
22 \\
23\end{array}$ & 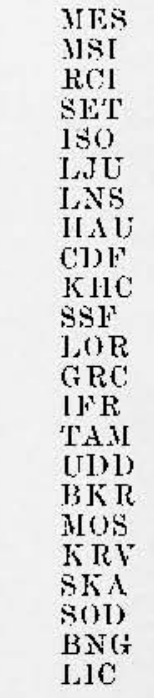 & 13 & $\begin{array}{l}11 \\
11 \\
11 \\
12 \\
12 \\
12 \\
12 \\
13 \\
13 \\
13 \\
13 \\
13 \\
13 \\
14 \\
14 \\
15 \\
15 \\
15 \\
16 \\
16 \\
16 \\
17 \\
17\end{array}$ & $\begin{array}{l}04,8 \\
04,5 \\
06,4 \\
03,1 \\
25,0 \\
34,0 \\
39,0 \\
13,6 \\
14,7 \\
15,0 \\
17,2 \\
17,5 \\
29,7 \\
08,0 \\
19,4 \\
27,7 \\
43,1 \\
49,0 \\
03,0 \\
03,0 \\
45,0 \\
13,1 \\
26,5\end{array}$ & $\begin{array}{r}2^{0}, 11055 \\
2^{0}, 11178 \\
2^{0}, 16551 \\
60,23554 \\
7^{0}, 76975 \\
8^{0}, 31991 \\
8^{0}, 73094 \\
11^{0}, 27501 \\
11^{0}, 36081 \\
110,332863 \\
11^{0}, 61456 \\
110,57938 \\
11^{0}, 94022 \\
15^{\circ}, 28640 \\
16^{0}, 26098 \\
22^{\circ}, 31069 \\
23^{0}, 77088 \\
24^{\circ}, 43947 \\
25^{0}, 194469 \\
25^{\circ}, 81265 \\
30^{\circ}, 61297 \\
33^{\circ}, 67546 \\
35^{\circ}, 42448\end{array}$ & $\begin{array}{l}-0,437 \\
-0,755 \\
\div 0,361 \\
-0,690 \\
-0,265 \\
-1,020 \\
-0,318 \\
+0,000 \\
-0,069 \\
+0,611 \\
-1,029 \\
-0,244 \\
-0,064 \\
-1,204 \\
+0,061 \\
-0,617 \\
+0,507 \\
-0,1063 \\
-0,402 \\
+0,827 \\
-0,567 \\
+0,794 \\
-0,844\end{array}$ \\
\hline \multicolumn{7}{|c|}{ Scossa $N .11$} \\
\hline $\begin{array}{l}1 \\
2 \\
3 \\
4 \\
5 \\
6 \\
5 \\
8 \\
9 \\
10 \\
11 \\
12 \\
13 \\
14 \\
15 \\
16 \\
17 \\
18\end{array}$ & 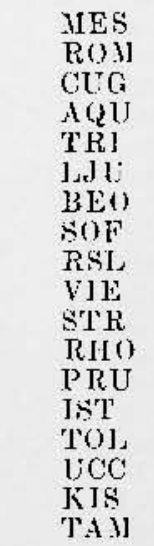 & 16 & $\begin{array}{l}43 \\
43 \\
43 \\
43 \\
44 \\
44 \\
44 \\
44 \\
45 \\
45 \\
45 \\
45 \\
45 \\
45 \\
45 \\
46 \\
46 \\
46\end{array}$ & $\begin{array}{l}18,3 \\
47,4 \\
49,0 \\
54,0 \\
40,0 \\
47,3 \\
55,7 \\
59,3 \\
00,0 \\
20,3 \\
29,0 \\
39,8 \\
40,0 \\
4 C, 0 \\
57,8 \\
09,0 \\
16,0 \\
34,4\end{array}$ & $\begin{array}{r}1^{0}, 99774 \\
4^{0}, 07254 \\
4^{0}, 20814 \\
4^{0}, 50813 \\
7^{0}, 87213 \\
8^{\circ}, 26187 \\
8^{0}, 90930 \\
9^{\circ}, 21485 \\
9^{\circ}, 18934 \\
10^{\circ}, 67902 \\
11^{\circ}, 40251 \\
12^{\circ}, 18142 \\
12^{\circ}, 18321 \\
12^{\circ}, 71378 \\
13^{\circ}, 49224 \\
14^{\circ}, 35310 \\
14^{\circ}, 81232 \\
16^{\circ}, 34680\end{array}$ & $\begin{array}{r}10,260 \\
-0,202 \\
-0,528 \\
-0,213 \\
-1,012 \\
+0,817 \\
0,246 \\
-0,369 \\
+0,683 \\
+0,483 \\
-0,614 \\
-0,367 \\
-0,191 \\
-1,307 \\
-0,250 \\
+0,131 \\
+1,118 \\
-0,199\end{array}$ \\
\hline
\end{tabular}




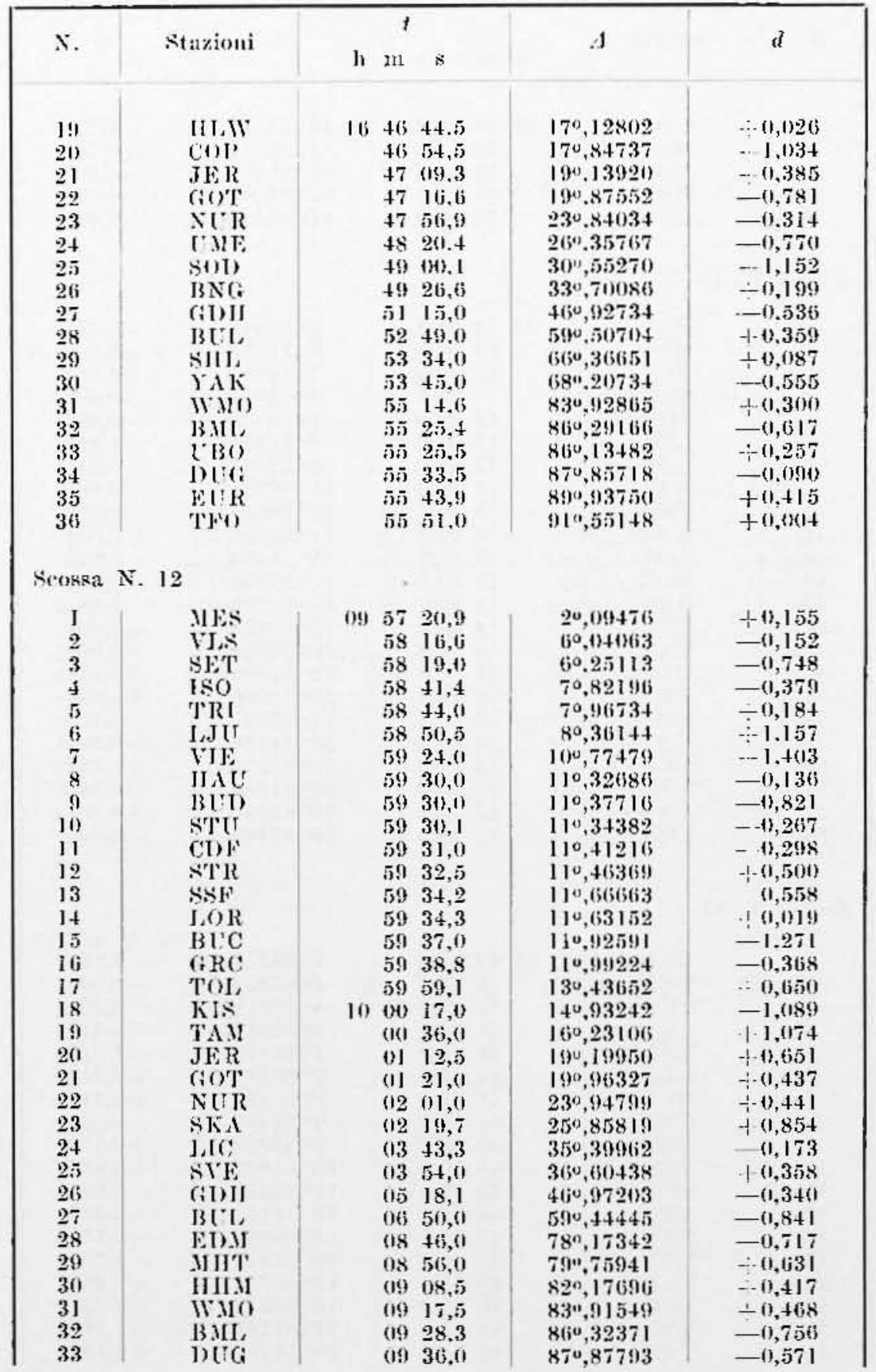




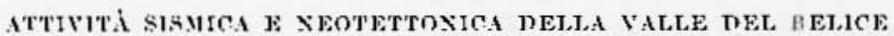

\begin{tabular}{|c|c|c|c|c|c|c|}
\hline \multirow{2}{*}{ s. } & \multirow{2}{*}{ Stazioni } & \multicolumn{3}{|c|}{1} & \multirow{2}{*}{1} & \multirow{2}{*}{$d$} \\
\hline & & lı & mi & $\mathrm{s}$ & & \\
\hline \multicolumn{7}{|c|}{ Scossa N. 13} \\
\hline l & MES & 14 & 36 & 05,1 & $2^{\circ}, 15728$ & $-0,3\{\}$ \\
\hline 2 & SE'T & & 37 & 02,0 & $6^{\circ} .19096$ & $-0, \mathrm{i}+5$ \\
\hline 3 & Ist) & & 37 & 25,8 & $7^{\circ}, 86148$ & -10.272 \\
\hline 4 & I.J1: & & 37 & 35,41 & $8^{\circ}, 4+310$ & $+0,779$ \\
\hline j) & MNY & & 37 & 43,0 & 90.07670 & $+01,112$ \\
\hline ij & $\mathrm{RSI}_{4}$ & & 37 & 46,0 & 90,27407 & - 10,305 \\
\hline 7 & KJJC & & 38 & 15,0 & $11^{0}, 45518$ & $-0,6009$ \\
\hline 8 & LBF & & 38 & 15,3 & $11^{0}, 39970$ & $-40,455$ \\
\hline 9 & ('J) l" & & 38 & $10 t, 3$ & 110,46010 & . $0, \overline{50} \mid$ \\
\hline 10 & SSF & & 38 & 18,3 & $11^{0}, 00510$ & $-(1,706$ \\
\hline 11 & $\mathrm{I}, 0 \mathrm{R}$ & & 38 & 18,8 & $11^{\circ},(5719)$ & $-(0,245$ \\
\hline 12 & Q \&R: & & 38 & 23,0 & $12^{n}, 02999$ & $-(0,403$ \\
\hline 13 & I'RU & & 38 & $2 \overline{7}, 0$ & $12^{0} .359+8$ & $-(0,8+0)$ \\
\hline 14 & NIE & & 38 & $3 \bar{n}, 6$ & 120,90144 & $\therefore 0,513$ \\
\hline 15 & $\mathrm{TOH}$ & & 38 & 42,0 & $13^{\circ}, 41127$ & $f(0.17)$ \\
\hline 16 & TAll & & 39 & 18,0 & $16^{0}, 1+256$ & $+0,5211$ \\
\hline 17 & $\mathrm{~J}$ IIN & & 40 & 39,6 & $23^{\circ}, 42551$ & $\div 0,505$ \\
\hline 18 & В K R & & 40 & 42,8 & $23^{\circ}, 82948$ & $-0,211$ \\
\hline 19 & Jis: & & 42 & 26,4 & $35^{\circ}, 31089$ & $\therefore 0,108$ \\
\hline 20 & HIIIII & & 47 & 52,1 & $82^{\circ}, 21682$ & $\therefore 0,290$ \\
\hline 21 & W.110 & & 48 & 01,1 & 830,90670 & \begin{tabular}{l|l}
$-0,593$ \\
$-0,5$
\end{tabular} \\
\hline 22 & DUE & & 48 & 18,10 & 870,82718 & $-1,233$ \\
\hline \multicolumn{7}{|c|}{ Senssa N. 14} \\
\hline I & MES & 16 & $2 \mathrm{i}$ & 36,0 & $\left.2^{\circ}, 08\right](j 3$ & $+0,453$ \\
\hline 2 & ROM & & 27 & $0+, 0$ & $4^{\circ}, 3+2169$ & $-0,899$ \\
\hline$\overline{3}$ & SE'T & & 27 & 34,5 & $6^{\circ}, 25424$ & 0,427 \\
\hline+ & MON & & 27 & 50,0 & 70,29179 & $\therefore \mathbf{0 , 6 + 8}$ \\
\hline 5 & SKo & & 27 & $5 \overline{3}, 2$ & $7^{0}, 74172$ & $-0,434$ \\
\hline (i & 150 & & 27 & 51,5 & $7^{\circ}, 81422$ & $-0,177$ \\
\hline 7 & 'TRJ & & 27 & 58,3 & 70,95302 & $-40,293$ \\
\hline 8 & MNY & & 28 & 14,0 & $9^{\circ}, 02872$ & $-1 .\langle, 452$ \\
\hline 9 & RSI. & & 28 & 17,0 & $9^{\circ}, 22207$ & $+0,784$ \\
\hline 10 & KIIC & & 28 & 46,0 & 110,36326 & $7 \cdot\{1,404$ \\
\hline iI & CDF & & 28 & 46,4 & 110,40290 & $\div 11,264$ \\
\hline 12 & $1, \mathrm{BF}$ & & 28 & 46,4 & $1] 0,35374$ & $+0,933$ \\
\hline 13 & SST & & 28 & 48,5 & $11^{0,66139}$ & $-1,1+9$ \\
\hline 14 & GRe & & 28 & 53,6 & 110,98695 & $-(1,461$ \\
\hline 15 & JER & & 30 & 27,0 & 390,19187 & $+0,389$ \\
\hline 16 & GO'T & & 3 & 35,0 & 190,94989 & $-0,270$ \\
\hline 17 & IIIN & & 31 & 09,2 & $23^{0}, 33816$ & $-0,285$ \\
\hline 18 & $\mathrm{BKR}$ & & $3 i$ & 13,6 & $23^{\circ}, 7+6992$ & $\begin{array}{r}+0,152 \\
+\end{array}$ \\
\hline is & 1'l & & 31 & 22,0 & $24^{\circ}, 167776$ & $-0,310$ \\
\hline 20 & BNE & & 32 & 42,5 & $33^{\circ}, 63778$ & $-\{0,63\}]$ \\
\hline 21 & I.IE: & & 32 & 58,6 & $35^{0}, 41681$ & $+(3,469$ \\
\hline 22 & IIIIXI & & 38 & 22.3 & $82^{\circ}, 17116$ & - 0,508 \\
\hline 23 & B.110 & & 38 & 44,1 & 860,31813 & $+0,329$ \\
\hline 24 & 1) $[: 6$ & & 38 & 51,7 & 870,87401 & $\therefore 0,+116$ \\
\hline
\end{tabular}


2. - Scontato che qualsiasi tentativo di correlazione fra sismicità e neotettonica di una regione non puó prescindere dalla conoscenza. della distribuzione spazio-temporale degli ipocentri, si è avviata, sulla base dej soli dati miorosismici, la determinazione analitica delle coordinate ipocentiali del maggrior numero possibile di scosse. Necessariamente scartati i metodi di determinazione che si avvalgono dei rilevamenti di un sufficiente numero di staxioni prossime all'epicentro - la stazione piu vicina all'area sede dei moti tellurici dista cirea $200 \mathrm{~km}$ - si è scelto un metorlo già largamente sperimentato che non presenta restrizione alcuna riguardo alle distanze epicentrali. Precisamente, il metodo statistico di faloi (4), ohe tradotto in linguasgrio macelina, la reso possibile l'impiego di un claboratore elettronico IBM 11-30. Ciù ha ovviamente facilitato di molto l'esecuzione dei calcoli, specialmente nella fase di selezione dei dati, consentento la riduzione delle incertezze statisticlic sui parametri ipocentrali entro liniti acestabili. Tenuto conto infatti della finalita delle determinazioni e la particolare considernzione dichiarata tla vari ricereatori per Ia geologia duIla zona coneussa, si è convenuto di scegliere cisseuno dei dati da elaborare, oltre che sulla base usuale dell'attendibilitid di ogni singola stazione, anche in relazione alla compatibilità di ciascuno di essi nell'ambito dell'insieme di dati ntilizzati in ogni singola determillazione.

I tempi di inizio delle $P$ (o $P n$ ) utilizzate dalla machlina $(A p)$ pendice I) sono guelli pubblicati nel bollettino mensile della Union Geodesique et Geoplyysique Internationale (B.C.I.S.), essendo del tutto insdeguato il numero di sismogrammi ricevuto dalle varie stazioni cui ne era sitata fatta richiesta.

Je determinazioni effettuate (Tabella II), complessivamente 14, sono relative a tutti i terremoti di magnituro $M \geqslant 4,4$ (Messina Univ.) verificatisi nella Sicilia centro-occislentale dal 14 Gennaio 1968 a tutt'oggi. Il valore 4,4 per lib magnitulo i risultato infatti valore soglia per un sorlilisfacente rilevamento strumentale in un rongruo numero di osservatori.

3. - Le profonlitic calcolate (Tab. II) presentano valori compresi fra 0 e $28 \mathrm{~km}$ e gli epicentri corrispondenti individnano un'area epicentrale rolativamente ristretta $\left(\sim 400 \mathrm{~km}^{2}\right)$ comprenciente Gibellina, Poggioreale, Salaparuta, Montevago, Partanna e S. Nargherita di Belice, rioi 6 degli 8 centri abitati maggiormente rovinati. Pertanto $i$ 14 terremoti più forti dell'intero periorlo sismico $(4,4 \leqslant M \leqslant 5,9)$, 
compresa la scossa più profonda (N. 11), avrebbero origine crostitle. Per le 6 scosse $1,5,6,12,13$ e 14 fra cui anche la scossa principale, totte con $h_{\max } \leqslant 12 \mathrm{~km}$, la stuperficialitì ipoeentrale determinata è poi tale dia far riguardare alla geologia della zona concussa con preminente interesse.

Di rletta zona pertanto, quasi a cavallo fra la Valle di Mazame la Valle del Belice, eosì come flelle aree immerliatamente circostanti, si ritiene opportuno riportare brevemente i seguenti lineamenti geomorfologici $\left(-, 3,1^{5}\right)$.

La Valle ti Mazara, incisa in un complesso ali argille, argille matnose, molasse ed episodi conglomeratici, ristulta di dne bacini idrogrufici distinti: Ja valle del fitme Fredilo ele sfocia nel Tirreno e la valle del fiume Delia che sbocot nel Canale di Sicilia. Il tutto costituisce nu'tampia depressione moderatamente ondulata.

Ia valle del Belice, sita a SE della precedente, è larga ed ondulata a $\mathrm{NE}$, si restringe sensibilmente con versanti ripiłi e scoscesi nel tratto intermedio e si allargn poi ntovamente fra pendici declivi nel tratto terminale. Le formazioni che vi affiorano presentano nna serie stratigrafica pressocché continua; la sucessione è greneralmente ordiuata da XVT a SE, dalla più antien alla più recente.

Le formazioni della Valle di Mazara sono attribnite al Miocene medio (15), la clorsale di A.te Finestrelle che supera i $650 \mathrm{~m}$ s.l.m. ì invece formata daIla "Serie gessoso solfifera"(1) del Miocene superiore, ricoperta da marne biancastre a Globigerine del Pliocene inferiore, estententisi principalmente sulle pendici a $\mathrm{S}$ della dorsale.

La valle del Belice, infine, presenta in prevalenza afforamenti di argille e argille sabbiose con intercalazioni di calcari detritico-organogeni e di sabbioni calctri cementati attribuibili al Pliocene. Fella parte a monte del versante orientale della Valle, sono poi visibili ricoperture di ealeari detritico-organogeni grossolani retti tuf enlearei.

J'elemento di principale interesse, in rapporto alla sismicitì riscontrata, sembra comunque essere costituito dalla esistenza di linee di giunzione fra tree to diversa evoluzione geologica.

L'allineamento Montevago-Contessa Entellina-Corleone, infatti, individua una frontiera a S e SE della quale i Monti Sicani e le loro propaggini oecirentali costituiscono una zona in massima parte emersa durante il Miocene ed il Pliocene $\left(^{2}\right)$. Per Cafiisch e Sclimidt di Friedberg ( $\left.{ }^{3}\right)$, in particolare, questa eatena di montagne è emersa o si trova in posizione rli alto strutturale sin dal Trias medio-superiore. 


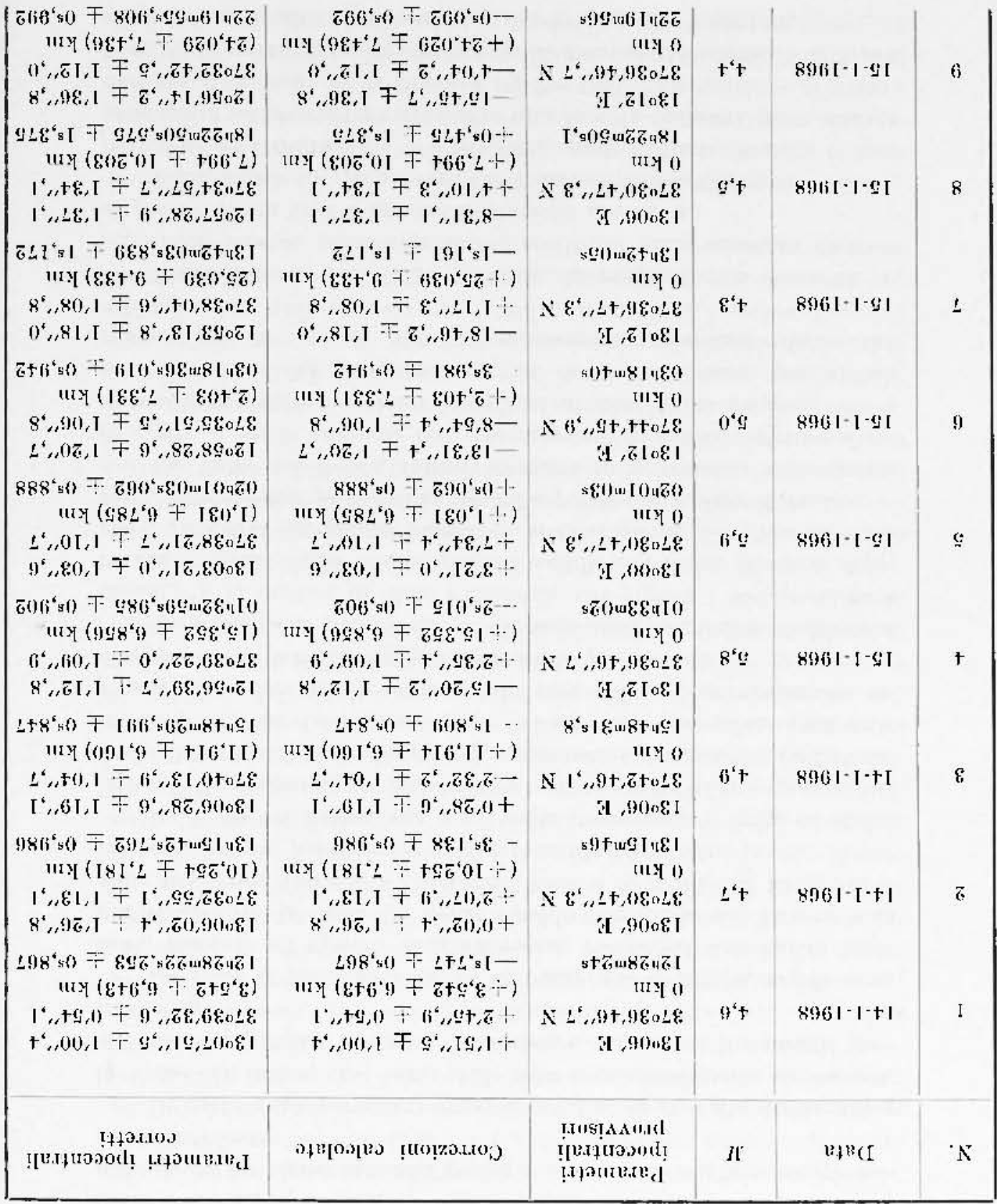

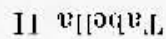




\begin{tabular}{|c|c|c|c|c|c|}
\hline N゙. & ])atal & II & $\begin{array}{l}\text { P'aramet ri } \\
\text { ipocentrati } \\
\text { provianori }\end{array}$ & Correzioni calcolate & $\begin{array}{c}\text { Paxametri ipocentrali } \\
\text { corretti }\end{array}$ \\
\hline 10 & $16 \cdot 1 \cdot 1908$ & 4,5 & 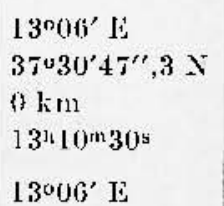 & $\begin{array}{l}-10^{\prime} 09^{\prime \prime}, 7 \pm 1^{\prime} 14^{\prime \prime}, 0 \\
-6^{\prime} 10^{\prime \prime}, 4 \pm 1^{\prime} 07^{\prime \prime}, 2 \\
(-15,670 \pm 9,390) \mathrm{km} \\
-0,100 \pm 1 \mathrm{~s}, 189 \\
-0^{\prime} 07^{\prime \prime}, 5 \pm 1^{\prime} 09^{\prime \prime}, 9\end{array}$ & $\begin{array}{l}12^{0} 55^{\prime} 50^{\prime \prime}, 2 \pm 1^{\prime} 14^{\prime \prime}, 0 \\
37^{\circ} 36^{\circ} 57^{\prime \prime}, 7 \pm 1^{\prime} 07^{\prime \prime}, 2 \\
(15,670 \pm 9,390) \mathrm{km} \\
\left.13^{11}\right] 0^{\mathrm{m} 29^{*}, 900} \pm 1^{8}, 189 \\
13^{\circ} 03^{\prime} 52^{\prime \prime}, 5 \pm 1^{\prime} 09^{\prime \prime}, 9\end{array}$ \\
\hline 11 & $16-1.1968$ & 5,8 & $\begin{array}{l}37^{0}+2^{\prime}+6^{\prime \prime}, 1 N \\
0 \mathrm{kIIt} \\
10^{\mathrm{h}}+2^{\mathrm{m}} 44^{\mathrm{s}}, 3\end{array}$ & $\begin{array}{l}-3^{\prime} 07^{\prime \prime}, 0 \pm 0^{\prime} 59^{\prime \prime}, 9 \\
(+27,734 \pm 5,303) \mathrm{km} \\
\therefore-15,230 \pm 0^{5}, 7+7\end{array}$ & $\begin{array}{l}37039^{\prime} 39^{\prime \prime}, 1 \pm 0^{\prime} 59^{\prime \prime}, 9 \\
(27,734 \pm 5,303) \mathrm{lm} \\
16^{1 \mathrm{~h}} 42^{\mathrm{n}} 45^{3}, 530 \div 0^{5}, 747\end{array}$ \\
\hline 12 & $25-1-1048$ & 5,6 & 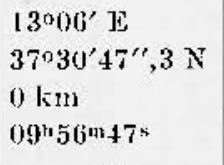 & $\begin{array}{l}-8^{\prime} 11^{\prime \prime}, 8 \pm 1^{\prime} 18^{\prime \prime}, 7 \\
1 \cdot 3^{\prime} 27^{\prime \prime}, 5 \pm 1^{\prime} 10^{\prime \prime}, 6 \\
(--0,320 \pm 0,589) \mathrm{km} \\
-2^{5}, 991 \pm(1,9), 906\end{array}$ & $\begin{array}{l}12057^{\prime} 48^{\prime \prime}, 2 \pm \mathrm{I}^{\prime} 18^{\prime \prime}, 7 \\
37^{\circ} 34^{\prime} 14^{\prime \prime}, 8 \pm \mathrm{I}^{\prime} 10^{\prime \prime}, 6 \\
(0,320 \pm 6,589) \mathrm{km} \\
09^{\mathrm{h}} 50^{\mathrm{m}}+6^{\mathrm{m}} 44^{\mathrm{s}}, 009 \pm 0 \mathrm{0}, 906\end{array}$ \\
\hline 13 & $25.1-1968$ & 4,7 & 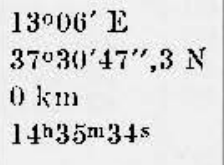 & $\begin{array}{l}-11^{\prime} 38^{\prime \prime}, 7 \pm 1^{\prime} 24^{\prime \prime}, 5 \\
-1^{\prime} 05^{\prime \prime}, 6 \pm 1^{\prime} 08^{\prime \prime}, 3 \\
(\div 4,857 \pm 7,279) \mathrm{km} \\
-3^{5}, 757 \pm 0^{5}, 974\end{array}$ & $\begin{array}{l}12^{\circ} 54^{\prime} 21^{\prime \prime}, 3+1^{\prime} 24^{\prime \prime}, 5 \\
37^{0} 29^{\prime} 39^{\prime \prime}, 7 \pm 1^{\prime} 08^{\prime \prime}, 3 \\
(4,857 \pm 7,279) \mathrm{km} \\
14^{11} 35^{n 12} 28^{5}, 243+0^{5}, 974\end{array}$ \\
\hline 14 & 12.2 .1968 & 4,6 & $\begin{array}{l}12^{\circ} 48^{\prime} \mathrm{E} \\
37^{0} 30^{\prime} 47^{\prime \prime}, 3 \mathrm{~N} \\
0 \mathrm{kIII} \\
16^{\prime \prime 2} 25^{\mathrm{m}} 59^{\mathrm{s}}\end{array}$ & $\begin{array}{l}10^{\prime} 35^{\prime \prime}, 0 \pm 1 \times 15^{\prime \prime}, 2 \\
4-4^{\prime} 16^{\prime \prime}, 1 \pm 1^{\prime} 13^{\prime \prime}, 5 \\
(+4,907 \pm 6,909) \mathrm{km} \\
+0 \times, 487 \pm 00^{5}, 953\end{array}$ & $\begin{array}{l}12^{\circ} 58^{\prime} 35^{\prime \prime}, 6 \pm 1^{\prime} 15^{\prime \prime}, 2 \\
37035^{\prime} 03^{\prime \prime}, 4 \pm 1^{\prime} 13^{\prime \prime}, 5 \\
(4,907 \pm 6,9) 99) \mathrm{km} \\
\left.16^{\prime \prime 2} 25^{\mathrm{m}} 59\right)^{5}, 487+05,953\end{array}$ \\
\hline
\end{tabular}

Sota - 1 valori riporlati per i parametri ipocentrali sono nellorline: Iongituline, latituline geocentrica, profonditia, tempo origine.

Ia Talle di Mazara, invece, e probabilmente buona javte flella Valle del Belice, disposte a $\mathrm{S}$ e $\mathrm{XW}$ dell'allineamento precisato, lamno subito una notevole subsirlenza de lat abbassato il tetto della serie stratigrafiea a jiù di $1000 \mathrm{~m}$ di profondità ( $\left.{ }^{3}\right)$.

Il bacino subsidente, colmato nel Yiocene merlio rla rlepositi argilloso-arenacei e successivamente dalla sovastante serie evaporition gessoso-sollifera, a partire dall'alto I'liocene inferiore sarebbe ala considerare seisso in due bacini subsidenti rlistinti, separati fla natorsale con andamento NE-SW riferibile in pration alla direttrice Castelvetrano-y.te linestrelle.

Riassumendo quindi, l'area macrosismicamente perturbata può essere suldivisa in tre zone contigre, delimitate dalle due linee di 
Appexide 11

\begin{tabular}{|c|c|c|c|c|c|c|}
\hline \multirow{2}{*}{ N. } & \multirow{2}{*}{ Stazioni } & \multicolumn{3}{|c|}{$t$} & \multirow{2}{*}{$\Delta$} & \multirow{2}{*}{$d$} \\
\hline & & $h_{1}$ & III & is & & \\
\hline \multicolumn{7}{|c|}{ Senssa N. I } \\
\hline 1 & MES & 12 & 28 & 57,1 & $1^{0}, 94966$ & $-0,582$ \\
\hline 2 & $\mathrm{TAR}$ & & 29 & 28,0 & $4^{0}, 15621$ & 0.037 \\
\hline 3 & VISS & & 29 & 53,0 & 50,90089 & $-0,350$ \\
\hline 4 & SET & & 29 & 59,0 & 60,39619 & $-0,676$ \\
\hline 5 & $\operatorname{sko}$ & & 30 & 16,1 & $7^{\circ}, 60869$ & $-0,564$ \\
\hline (i & TRI & & 30 & 19,5 & $7^{\circ}, 82186$ & $-0,148$ \\
\hline 7 & J.JU & & 30 & 26,0 & $8^{\circ}, 27466$ & $-0,001$ \\
\hline 8 & $\Lambda \mathrm{TL}$ & & 30 & 27,0 & $8^{\circ}, 370557$ & $-0,3+1$ \\
\hline 9 & JNS & & 30 & 33,3 & $8^{\circ}, 78742$ & $-1-0,186$ \\
\hline 10 & STE: & & 31 & 07,3 & $11^{\circ}, 30507$ & $-0,410$ \\
\hline 11 & S'I'R & & 3] & 09,5 & $11^{0}, 43737$ & $-0,013$ \\
\hline 12 & TOL & & 3] & 38,2 & $13^{0}, 54823$ & $+0,403$ \\
\hline 13 & TAM & & 32 & 15,0 & $\left.16^{\circ}, 3522\right]$ & $-1-0,656$ \\
\hline 14 & OUJ, & & 34 & 19,0 & $28^{\circ}, 31410$ & $-0,171$ \\
\hline 15 & SOJ) & & 34 & 39,0 & $30^{\mathrm{v}}, 55972$ & $-0,275$ \\
\hline $16 j$ & $\mathrm{~J} 3 \mathrm{NG}$ & & 35 & 05,7 & $33^{\circ}, 67274$ & $-0,805$ \\
\hline 17 & LIC & & 35 & 22,8 & $35^{\circ}, 53027$ & 10,333 \\
\hline 18 & WHO & & 40 & 55,3 & $83^{\circ}, 98171$ & $\cdot-0,048$ \\
\hline \multicolumn{7}{|c|}{ Scossa N. 2} \\
\hline 1 & MES & 13 & 16 & 16,7 & $2^{v}, 00257$ & $-0,213$ \\
\hline 2 & Rosl & & iti & $47, \overline{1}$ & $4^{\circ}, 200001$ & $-0,716$ \\
\hline 3 & AQLi & & $16 i$ & $5 \overline{5}, 0$ & $4^{0},(63246)$ & $-10,645$ \\
\hline 4 & VLS & & 17 & 12,8 & $5^{0}, 94173$ & $-0,0 \overline{5} 5$ \\
\hline 5 & SET & & 17 & 18,5 & $\mathrm{t}^{\circ}, 34522$ & $-0,08 \overline{0}$ \\
\hline 6 & $\mathrm{SKO}$ & & 17 & 37,1 & $7^{0}, 69491$ & $-0,402$ \\
\hline 7 & TRI & & 17 & 43,0 & $7^{0}, 99622$ & $+1,277$ \\
\hline 8 & $\mathrm{I}\lrcorner \mathrm{JU}$ & & 17 & 47,0 & $8^{\circ}, 38382$ & $-0,158$ \\
\hline 9 & ATL & & 17 & 47,5 & $8^{\circ}, 4085 i$ & $-0,003$ \\
\hline 10 & JNS & & 17 & 54,0 & $8^{0}, 860506$ & $-1-0,184$ \\
\hline 11 & STU & & 18 & 29,4 & $11^{0}, 40182$ & $-1-0,740$ \\
\hline 12 & $\mathrm{KHC}$ & & 18 & 29,5 & $11^{0}, 40950$ & $-10,735$ \\
\hline 13 & NITR & & 20 & 57,9 & $23^{\circ}, 05718$ & $-0,200$ \\
\hline 14 & Mos & & 21 & 03,0 & $24^{\circ}, 43236$ & $\cdot \mid \cdot 0,204$ \\
\hline 15 & TAB & & 21 & 20,0 & $26^{\circ}, 15330$ & $+0,000$ \\
\hline 16 & INAE & & 21 & 20,6 & $26^{\circ}, 47953$ & $-1,504$ \\
\hline 17 & KJR & & 21 & 57,0 & $30^{\circ}, 44809$ & $-0,924$ \\
\hline 18 & $\mathrm{BNG}$ & & 22 & 25,7 & $33^{\circ}, 57298$ & $+0,431$ \\
\hline 19 & JIC & & 22 & 41,5 & $35^{0}, 42450$ & $+0,30 \mathrm{I}$ \\
\hline 20 & BTL & & 25 & 48,0 & $59^{\circ}, 37997$ & $\therefore 0,088$ \\
\hline 21 & TIK & & 20 & 06,0 & 620,00172 & $-1), 185$ \\
\hline 22 & $B 07$ & & 28 & 11,0 & $83^{\circ}, 29833$ & $-0,409$ \\
\hline 23 & $B M O$ & & 28 & 26,4 & $86^{\circ}, 40963$ & $-0,599$ \\
\hline \multicolumn{7}{|c|}{ Scossa N. 3} \\
\hline I & MES & 15 & 49 & 03,2 & $1^{0}, 97284$ & $-0,186$ \\
\hline 2 & ROAL & & 49 & 32,8 & $4^{0}, 08471$ & $-0,681$ \\
\hline 3 & $\mathrm{AQU}$ & & 49 & 41,0 & $4^{0}, 51606$ & $\dashv-1,394$ \\
\hline
\end{tabular}




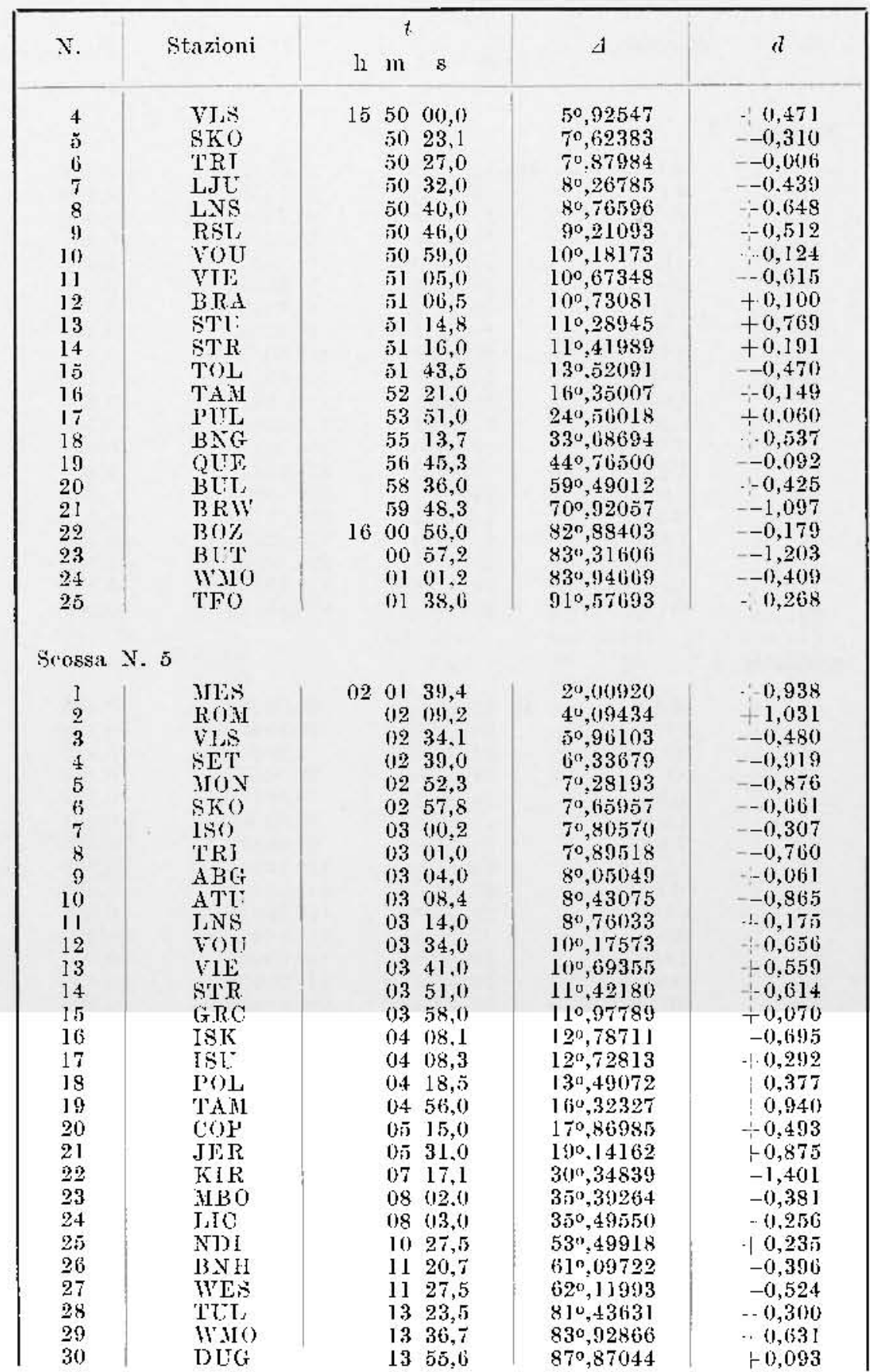




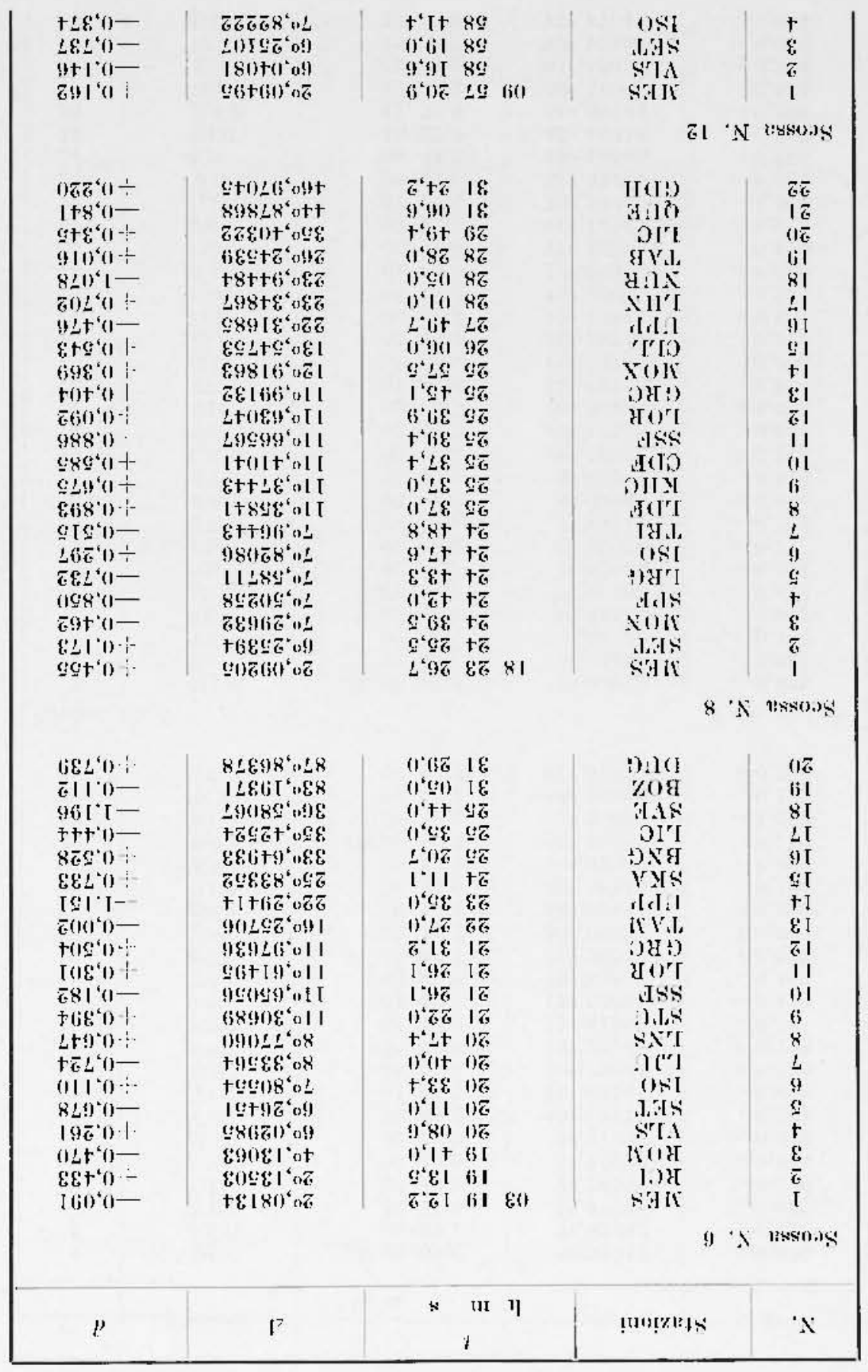




\begin{tabular}{|c|c|c|c|c|c|c|}
\hline \multirow{2}{*}{$x$} & \multirow{2}{*}{ Stazioni } & \multicolumn{3}{|c|}{$t$} & \multirow{2}{*}{$y$} & \multirow{2}{*}{$d$} \\
\hline & & $h_{1}$ & 111 & s & & \\
\hline 5 & TRI & 109 & 58 & $4+, 0$ & $7^{\circ}, 96708$ & $\therefore 0,188$ \\
\hline 6) & $\mathrm{L}, \mathrm{J} \mathrm{I}^{\top}$ & & $58:$ & 511,5 & 80,36180 & $-1,160$ \\
\hline 7 & VIL & & $59:$ & 24,0 & 1010.75522 & -1.4116 \\
\hline 8 & $11 \mathrm{Al}$ & & $59:$ & 311,0 & 110,32725 & $-0,134$ \\
\hline 9 & [3 [1]) & & 59 ; & $3 \&, 0$ & $11^{0}, 37760$ & $-0,820$ \\
\hline 10 & STI & & 59 : & 30,1 & $110,3+424$ & -0.265 \\
\hline il & CloF & & $59 ;$ & 31,0 & $11^{0}, 41257$ & -0.296 \\
\hline 12 & S'T'R & & $59:$ & 32,5 & $11^{0}, 46+119$ & $\therefore 0,502$ \\
\hline 13 & SSF & & $59:$ & 34,2 & $11^{\circ}$, bitios & $-0,556$ \\
\hline 14 & $\mathrm{~J}, \mathrm{OR}$ & & $50 !$ & 34,3 & $11^{0}, 63188$ & $--(1,1) 21$ \\
\hline 15 & BCTC: & & $59 !$ & 37,0 & $11 ", 92626$ & $-1,269$ \\
\hline 16 & $\because R C$ & & 5) : & 38,8 & 110,999260 & $-11,365$ \\
\hline 17 & $\mathrm{TOL}$ & & 5!) : & 59.1 & $13^{\circ}, 43634$ & - 0.6599 \\
\hline 18 & Kls & 10 & (1)) & {$[7,6)$} & $14^{\circ}, 932+7$ & -1.083 \\
\hline 19 & TAMI & & 190): & 36,6 & $16^{\circ}, 23066$ & 1,084 \\
\hline 20 & JER & & (1)) & 12,5 & $16 m^{m}, 19936$ & $+6,6553$ \\
\hline 21 & (iot' & & $01:$ & 21,11 & $160^{\circ}, 96358$ & $-0,433$ \\
\hline 22 & NLR & & $102 i$ & (11, & $23^{\circ}, 94840$ & 0.436 \\
\hline 23 & $S K A$ & & 172 & 19,7 & $25^{\circ}, 85869$ & $0.84 !$ \\
\hline 24 & $\mathrm{LIC}$ & & 03. & $+3,3$ & $35^{\circ}, 39940$ & -0.174 \\
\hline $2 \pi$ & SVl: & & $03:$ & $54,1)$ & $366^{\circ}, 60482$ & $-11,351$ \\
\hline 26 & (:1) H & & 05 & 18,1 & $4(61,(17220)$ & $\{1,345$ \\
\hline 27 & B $3 \mathrm{LI}$ & & $06:$ & $5(1,1)$ & $599^{\circ} .44421$ & $-\{1,845$ \\
\hline 28 & E13AI & & 108 & 46,41 & 780,173660 & $-1,724$ \\
\hline 29 & IIJ'T & & $08:$ & 56,0 & 790,75974 & $-1), 623$ \\
\hline 34 & [I JI3] & & 00 & 418,5 & 82,17731 & $-40,409$ \\
\hline 31 & WNo & & 09 & 17,5 & $83^{0} .91566$ & $-60,460$ \\
\hline 32 & B.110 & & 09 & 28,3 & $86^{\circ}, 32+16$ & $\therefore-1), \overline{7} 6 \bar{i}$ \\
\hline 33 & $10 \mathrm{CO}$ & & 199 & 36,0 & $87^{0}, 87844$ & $-11, \overline{3} 82$ \\
\hline \multicolumn{7}{|c|}{ Scossa N. 13} \\
\hline 1 & MES & 14 & $36) 1$ & 105,1 & $2^{\circ}, 15647$ & $-\{t, 160$ \\
\hline 2 & $\triangle \mathrm{ET}$ & & 37 & 02,0 & $\left(0^{\circ}, 19209\right.$ & $-11,538$ \\
\hline 3 & 150 & & 37 & 25,8 & 70,84731 & $\{1,245$ \\
\hline 4 & 1.J L & & $3 \bar{t}:$ & 35,0 & $80,+4821$ & 41,816 \\
\hline 5 & MNY & & 37 & 43,0 & 90,07649 & $+\{1,140$ \\
\hline (j) & RSL & & 37 & $4(;, 0)$ & $\left\{9^{\circ}, 27996\right.$ & $-10,333$ \\
\hline$i$ & KHC & & 38 & 15,0 & $11^{0}, 460.35$ & - 6,58 ! \\
\hline 8 & L.AB] & & 38 & 15,3 & $11^{0}, 40490$ & $-11,+439$ \\
\hline 9 & ('D) F & & 38 & 16,3 & $110,+75001$ & $\mid 1,, 514$ \\
\hline 10 & $\therefore S T$ & & 38 & 18,3 & $|10,7| 09 \mid$ & $-0,691$ \\
\hline 11 & $\mathrm{LOR}$ & & 38 & 18,8 & $11^{0}, 67775$ & $-10,259$ \\
\hline 12 & $\mathrm{ARC}$ & & 38 & 23,0 & 120,013576 & $-0,388$ \\
\hline 13 & l'RLI & & 38 & 27,11 & $120.36+73$ & $-0,817$ \\
\hline 14 & NIE. & & 38 & 35,6 & 120,965165 & : $\quad 0, ; i$ \\
\hline 15 & $\mathrm{TOH}_{4}$ & & 38 & 42.11 & $13^{0},+1+85$ & $\because 10,203$ \\
\hline 16 & TAM & & $3 !$ & 18,4 & $\left.16^{n}, 13851\right)$ & $-0,021$ \\
\hline 17 & LII N & & 40 & 39,6 & 230,43167 & $-0,+13$ \\
\hline 18 & $1 \mathrm{KR}$ & & 40. & $+2,8$ & $23^{0}, 82915$ & $-0,2+6$ \\
\hline 19 & $1,1 \mathrm{C}$ & & +2 & 26,4 & $35^{n}, 310745$ & $+11,066$ \\
\hline 20) & II HI.AI & & $47 ?$ & 52,1 & $82^{\circ}, 22264$ & $-0,133$ \\
\hline 21 & IVII & & 48 & 01.1 & $83^{\circ}, 01193$ & $\therefore 0,440$ \\
\hline 22 & $1) 10$ & & 48 & 18,6 & 870,83287 & $\cdots 1,+(13$ \\
\hline
\end{tabular}




\begin{tabular}{|c|c|c|c|c|c|c|}
\hline \multirow{2}{*}{ N. } & \multirow{2}{*}{ Stazioni } & \multicolumn{3}{|c|}{$t$} & \multirow{2}{*}{$\Delta$} & \multirow{2}{*}{$d$} \\
\hline & & lt & in & s & & \\
\hline \multicolumn{7}{|c|}{ Scossa X. 14} \\
\hline ] & \$ES & 16 & 26 & 36,0 & 20,08235 & $\div 0,588$ \\
\hline 2 & ROAL & & 25 & 04,0 & $4^{\prime \prime}, 1+972$ & $-01,847$ \\
\hline 3 & SET & & 27 & $34, \overline{5}$ & $6^{n}, 26362$ & $-0,272$ \\
\hline 4 & $310 \mathrm{~N}$ & & 27 & 50,0 & 70,29863 & $+0,698$ \\
\hline 5 & Sixo & & 27 & 55,2 & 70,74532 & $-0,363$ \\
\hline 6 & ISO & & 27 & $56, \overline{5}$ & $7^{\circ}, 82309$ & $-0,142$ \\
\hline 7 & TRI & & 27 & 58,3 & $7^{\circ}, 96050$ & $-0,267$ \\
\hline 8 & IINY & & 28 & 14,0 & $9^{0}, 03547$ & $+11,490$ \\
\hline 9 & RSI, & & 28 & 17,0 & $9^{\circ}, 22916$ & $+11,817$ \\
\hline 10 & KIIC: & & 28 & 46,0 & $1]^{\circ}, 37080$ & $+0,417$ \\
\hline 11 & ('DF' & & 28 & 46,4 & $11^{\circ}, 41036$ & $-0,27 \mathrm{~B}$ \\
\hline 12 & J.JPF & & 28 & 46,4 & $11^{\circ}, 36062$ & $.0,950$ \\
\hline $1 \overline{3}$ & $\triangle S F^{\circ}$ & & 28 & 48,5 & $11^{0}, 66802$ & -1.125 \\
\hline 14 & GRC & & 28 & i) 3,6 & 110,99375 & -0.437 \\
\hline 15 & $J E R$ & & 30 & 27,6 & $19^{\circ}, 18940$ & $+0,415$ \\
\hline 16 & $\mathrm{GOT}$ & & 30 & $3 \overline{5}, 0$ & 190,95748 & -0.351 \\
\hline 17 & IIIX & & 31 & $(19,2$ & $23^{\circ}, 34577$ & $-0,37 \pi$ \\
\hline 18 & $\mathrm{BKK}$ & & 31 & 13,6 & $23^{\circ}, 748+9$ & $+0,119$ \\
\hline 19 & l'le & & 31 & 22,0 & $24^{\circ}, 60444$ & $-0,393$ \\
\hline 20 & $\mathrm{BNCA}$ & & 32 & 42,5 & $33^{\circ}, 63018$ & $--0,6112$ \\
\hline 21 & $\mathrm{~L} / \mathrm{IC}$ & & 32 & 58,9 & $35^{\circ}, 41091$ & $-0,472$ \\
\hline 22 & 1] HसL & & 38 & 22,3 & $82^{\circ}, 17807$ & $-0,6531$ \\
\hline 23 & $B \times[0$ & & 38 & 44,1 & $80^{n}, 32501$ & -0.173 \\
\hline 24 & DIG & & 38 & 51.7 & $87^{n}, 88054$ & $-0,252$ \\
\hline
\end{tabular}

giunzione sopra specilicate ed indicate in Fig. 1 rispettivamente con $\mathbf{I}_{2}$ e $\mathbf{I}_{\mathbf{1}}$.

Premesso cio, ì possibile stabilire alcuni elementi di correlazione fra l'attivitì sismica e la tettonica della zona concussa.

Rilevato infatti che gli epicentri calcolati ed indieati nelia mapja di Fig. 2 presentano una distribuzione pressocché rettilinea, da NE verso SW, su un fronte di ciren $30 \mathrm{~km}$, e con l'estremo di NE in corrispondenza di Rocamena e I'estremo di SW a S di Partanna, risulta che detta disposizione epicentrale è sensibilmente parallela alla congimugente Castelvetrano-M.te Finestrelle e si discosta da questa di circa $4-5 \mathrm{~km}$ in direzione $\mathrm{E}$.

I soli epicentri dei terremoti 2,4 e $i$ non seguono l'andamento generale: il $\mathbf{X} .2$ i praticumente sulla congiungente Montevago-Contessa IEntellina $\left(\mathbf{I}_{2}\right)$, mentre il $\mathrm{N} . \pm \mathrm{e}$ il $\mathrm{N}$. $\tau$ sono ar $\mathrm{W}$ della direttrice Castelvetrano-Hl.te Finestrelle $\left(\mathbf{I}_{1}\right)$ (Fig. 3). 


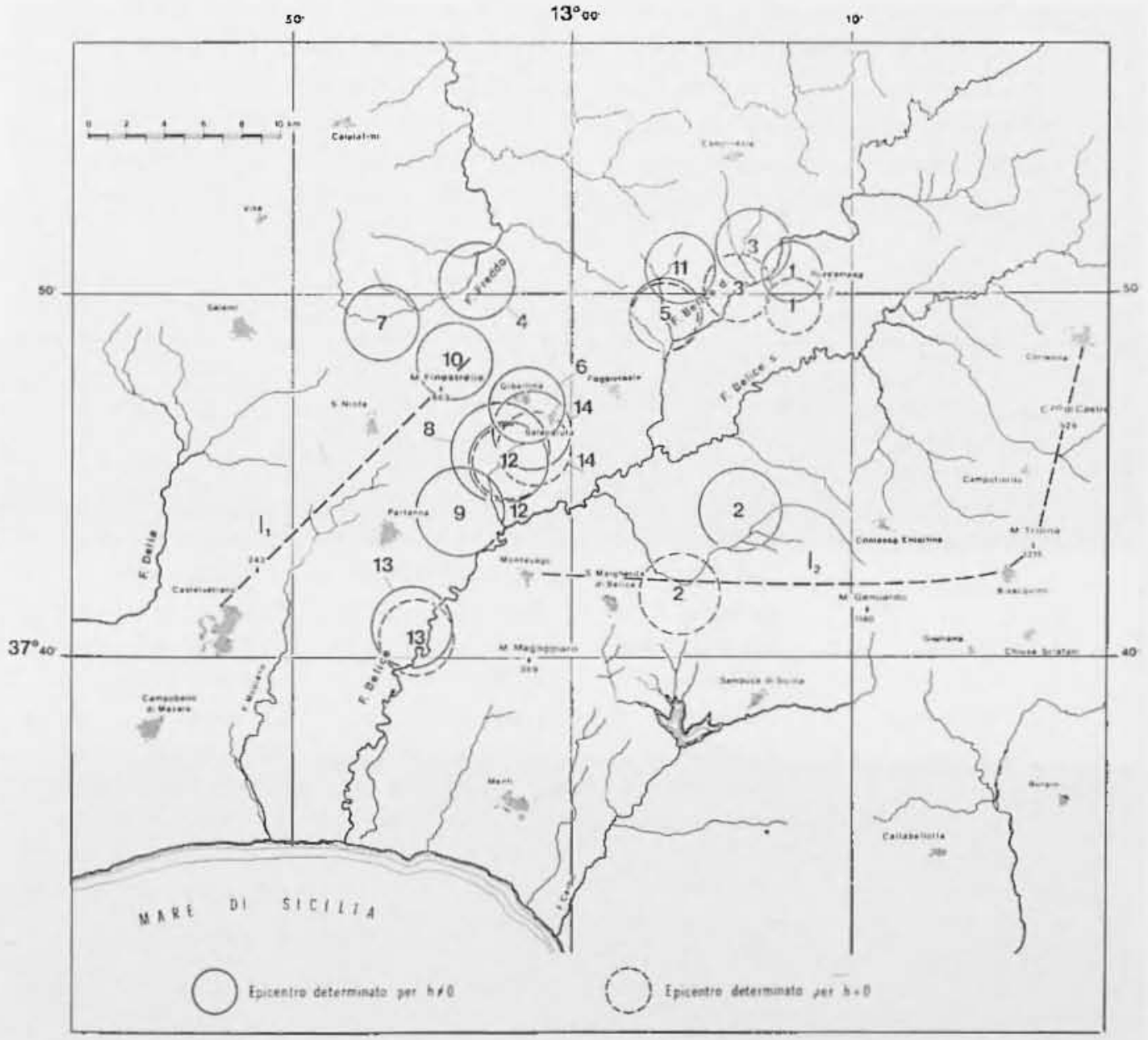

Fig. 2

Ritenendo questi primi risultati di un certo interesse e rilevando d'altra parte cle, in senso fisico, è scarsamente indicativo considerare profonditì ipocentrali di $3-4 \mathrm{~km}$ allorehe le incertezze ussociate sono merliamente di $\mathbf{i} \mathrm{km}$, si è convenuto di ripetere le determinazioni dei parametri ipocentrali, imponendo la condizione dii superficialità $h-0$, per tutte quelle scosse per le quali le precedenti determinazioni avevano indiento $h_{\text {max }} \leqslant 12 \mathrm{~km}$.

La nuova serie di determinazioni (Tab. III e Appendice II) non modifica apprezzabilmente la distribuzione epieentrale precedentemen- 
1e Nabilila (Fig. 2). Questa circostanza, unitamente a quanto già rilevato ed in ngginnta al carattere delle registarioni ottenute a Messina Univ. (Figg. 3, 4, 5, (i) sulle quali si vuole orn richimare l'atten\%ione, ronforta e la superticialitia e la distribuzione tegli ipocentri inizialneme: leteminati. A riguardo \& ta sotiolineare come terisivo, ai fini tlella prima Iocalizzazione, l'ajporto derivante talla ntilizzazione tei tempi

Tabella III

\begin{tabular}{|c|c|c|c|c|c|}
\hline N. & [):a1 a & $M$ & $\begin{array}{l}\text { parametri } \\
\text { ipocentrali } \\
\text { provvisari }\end{array}$ & Correzioni calcolate & $\begin{array}{c}\text { Parametri ipucentrali } \\
\text { corretti }\end{array}$ \\
\hline 1 & $14-1-1968$ & 4,6 & $\begin{array}{l}13^{\circ} 06^{\prime} \mathrm{E} \\
37^{\circ} 36^{\prime}+6^{\prime \prime}, 7 \mathrm{~V} \\
12^{\prime \prime 2} 28^{\prime n} 24^{\circ} \\
13^{\circ} 116^{\prime} \mathrm{E}\end{array}$ & $\begin{array}{l}+1^{\prime} 53^{\prime \prime}, 0 \pm 0^{\prime} 56^{\prime \prime}, 6 \\
+1^{\prime}+2^{\prime \prime}, 7 \pm\left(1^{\prime} 49^{\prime \prime}, 3\right. \\
-2^{\mathrm{n}}, 1 \overline{6} 6 \pm 0,123 \\
-0^{\prime} 21^{\prime \prime}, 4 \pm 1^{\prime} 29^{\prime \prime}, 0\end{array}$ & $\begin{array}{l}13^{\prime \prime}\left(17^{\prime} 52^{\prime \prime}, 9=\left(0^{\prime} 56^{\prime \prime}, 6\right.\right. \\
37038^{\prime} 29^{\prime \prime}, 4 \leq 0^{\prime} 49^{\prime \prime}, 3 \\
122^{\prime \prime 2} 8^{m} 21^{9}, 8+4=0^{9}, 123 \\
13005^{\prime} 38^{\prime \prime}, 5+1^{\prime} 29^{\prime \prime}, 0\end{array}$ \\
\hline 2 & 14.1 .1968 & 4.7 & $\begin{array}{l}37^{v} 30^{\prime}+7^{\prime \prime}, 3 \mathrm{~N} \\
\left.13^{\mathrm{n}}\right] 5^{\mathrm{m}} 463^{3}\end{array}$ & $\begin{array}{l}1^{\prime} 19^{\prime \prime}, 1 \doteq 1^{\prime} 07^{\prime \prime}, 16 \\
-4^{8}, 524+10^{\circ}, 178\end{array}$ & 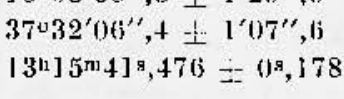 \\
\hline 3 & 14.1 .1968 & 4,9 & $\begin{array}{l}\left.13^{\circ} 006^{\prime}\right] \mathrm{i} \\
\left.37^{\circ}+2^{\prime} 46^{\prime \prime},\right] \mathrm{N} \\
35^{\mathrm{h}} 48^{\mathrm{m}} 31^{\mathrm{s}}, 8\end{array}$ & $\begin{array}{l}-0^{\prime} 03^{\prime \prime}, 8 \div J^{\prime} 23^{\prime \prime}, 1 \\
-3^{\prime} 40^{\prime \prime}, 8 \pm 0^{\prime} 58^{\prime \prime}, 1 \\
-3,422 \pm 0^{9}, 158\end{array}$ & $\begin{array}{l}13^{\circ} 05^{\prime} 56^{\prime \prime}, 2 \pm 1^{\prime} 23^{\prime \prime}, 1 \\
37^{\circ} 39^{\prime} 05^{\prime \prime}, 3 \pm 0^{\prime} 58^{\prime \prime}, 1 \\
15^{14} 48^{m} 28^{\circ}, 378+0^{\circ}, 158\end{array}$ \\
\hline 5 & $15-1-19068$ & 5,9 & $\begin{array}{l}13^{\circ} 00^{\prime} \mathrm{l} \\
37^{0} 30^{\prime} 47^{\prime \prime}, 3 \mathrm{~N} \\
02^{\prime \prime} 0 \operatorname{lm}^{\mathrm{m}\left(13^{5} .0\right.}\end{array}$ & $\begin{array}{l}\left.+3^{\prime}\right] 9^{\prime \prime}, 7 \pm 1^{\prime} 03^{\prime \prime}, 0 \\
+7^{\prime} 30^{\prime \prime}, 3 \pm 1^{\prime} 05^{\prime \prime}, 2 \\
-0 \mathrm{~s}, 071 \pm 0,15]\end{array}$ & 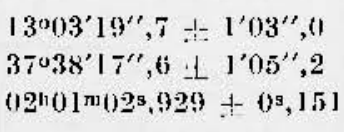 \\
\hline ij & $15-1-1968$ & $5,1)$ & $\begin{array}{l}\left.13^{\circ}\right] 2^{\prime} \mathrm{E} \\
37^{\circ} 44^{\prime}+6^{\prime \prime}, 5 \mathrm{~N} \\
63^{\mathrm{h}} 18^{\mathrm{m}}+0 \mathrm{0s}\end{array}$ & $\begin{array}{l}-133^{\prime} 34^{\prime \prime}, 4 \pm J^{\prime} 20^{\prime \prime}, 4 \\
-9^{\prime} 0 I^{\prime \prime}, 8 \pm 1^{\prime}\left(13^{\prime \prime}, 6\right. \\
-4^{3}, 285 \pm 0^{\mathrm{s}}, 164\end{array}$ & 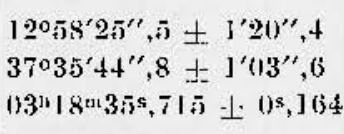 \\
\hline 8 & $15-1-1968$ & 4.5 & $\begin{array}{l}\left.13^{\circ} 0\right) 6^{\prime} \mathrm{F} \\
37^{\circ} 30^{\prime}+7^{\prime \prime}, 3 \mathrm{~N} \\
18^{n} 22^{m} 50^{\mathrm{s}}, 1\end{array}$ & $\begin{array}{l}8^{\prime} 01^{\prime \prime}, 7 \pm 1^{\prime} 30^{\prime \prime}, 9 \\
+3^{\prime} 30^{\prime \prime}, 9 \pm 1^{\prime} 25^{\prime \prime}, 1 \\
-0^{\mathrm{s}}, 580^{\prime} \pm(15,240\end{array}$ & 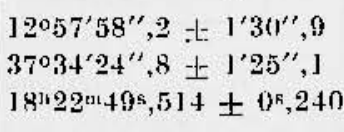 \\
\hline 12 & $25 \cdot 1 \cdot 3968$ & 50,0 & 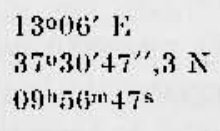 & $\begin{array}{l}-8^{\prime} 12^{\prime \prime}, 0 \pm 1^{\prime} 18^{\prime \prime}, \overline{5} \\
3^{\prime} 2 \overline{5}^{\prime \prime}, 8 \pm I^{\prime}\left(04^{\prime \prime}, 5\right. \\
3^{\mathrm{s}}, 035 \pm(1 \mathrm{~s}, 11 \mathrm{j} 6\end{array}$ & 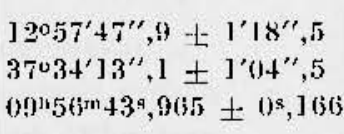 \\
\hline 13 & $25-1-1968$ & 4,7 & $\begin{array}{l}33^{\circ}\left(11 \mathrm{i}^{\prime} \mathrm{E}\right. \\
37^{\circ 0} 30^{\prime}+7^{\prime \prime}, 3 \mathrm{~N} \\
14^{\prime \prime} 35^{\mathrm{m}} 34^{\mathrm{s}}\end{array}$ & $\begin{array}{l}\left.-11^{\prime} 28^{\prime \prime},+\frac{1}{-1}\right]^{\prime} 23^{\prime \prime}, 9 \\
-1^{\prime} 27^{\prime \prime}, 2 \pm 1^{\prime}\left(12^{\prime \prime}, 3\right. \\
-(9,396 \pm 19,1816\end{array}$ & $\begin{array}{l}12054^{\prime} 31^{\prime \prime}, 6+1^{\prime} 23^{\prime \prime}, 9 \\
37029^{\prime} 20^{\prime \prime}, 1+1^{\prime}\left(12^{\prime \prime}, 3\right. \\
14^{\prime \prime} 35^{m} 27^{s}, 604+0^{s}, 186\end{array}$ \\
\hline 14 & $12.2-1968$ & $4.6 i$ & $\begin{array}{l}12^{\circ} 48^{\prime} \mathrm{L} \\
37^{\circ} 30^{\prime}+7^{\prime \prime}, 3 \mathrm{~N} \\
16^{\prime \prime} 25^{\mathrm{m}} \mathrm{m} 9^{\mathrm{s}}\end{array}$ & $\begin{array}{l}-10^{\prime} 4\left(1^{\prime \prime}, 0 \pm 1^{\prime}\right] 5^{\prime \prime}, 7 \\
-3^{\prime} 48^{\prime \prime}, 8 \pm 1^{\prime}\left(13^{\prime \prime}, 4\right. \\
-10 \%, 179 \pm(15,167\end{array}$ & 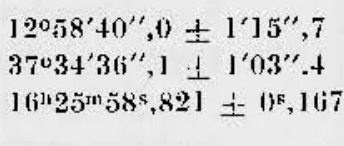 \\
\hline
\end{tabular}



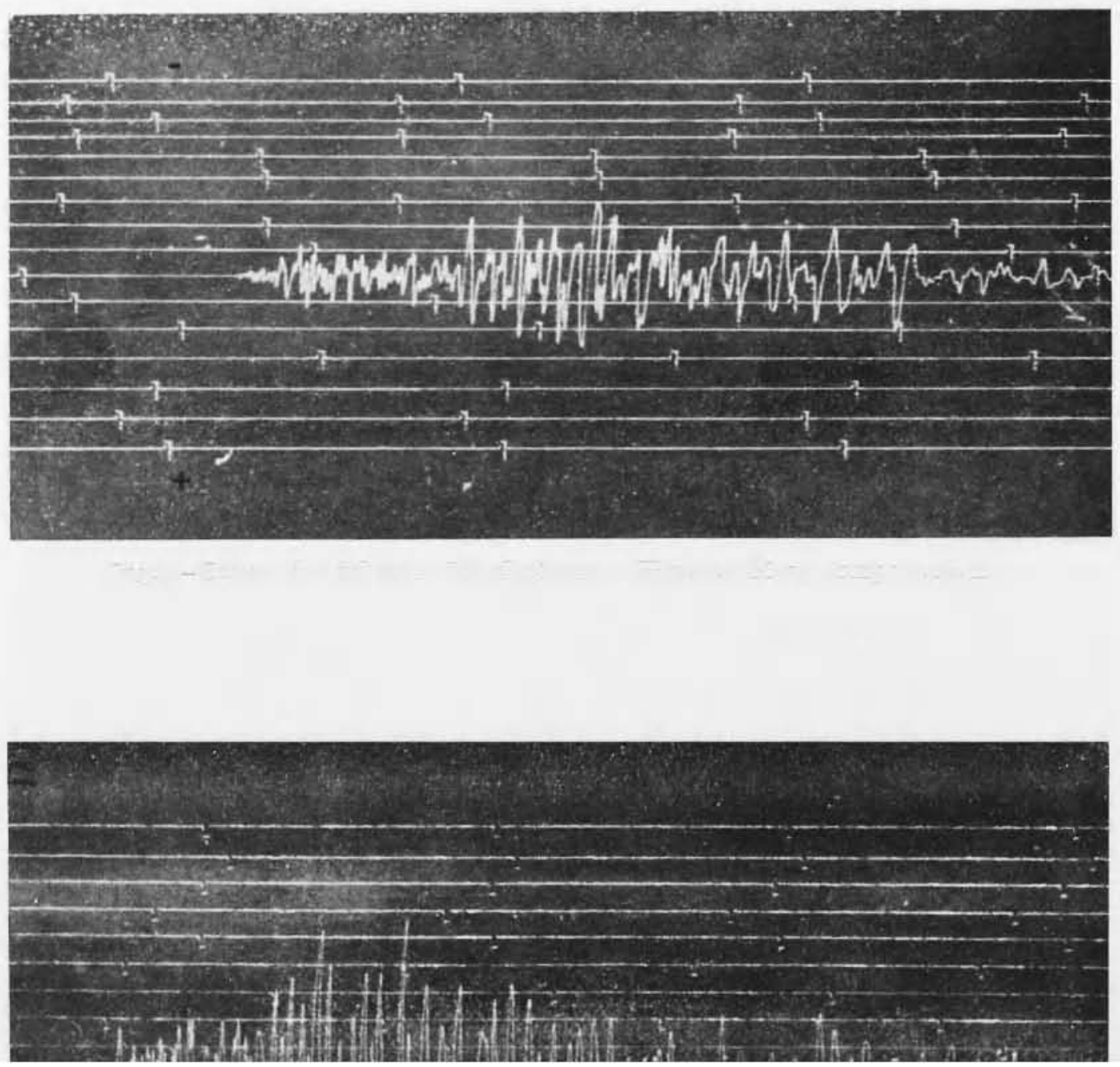


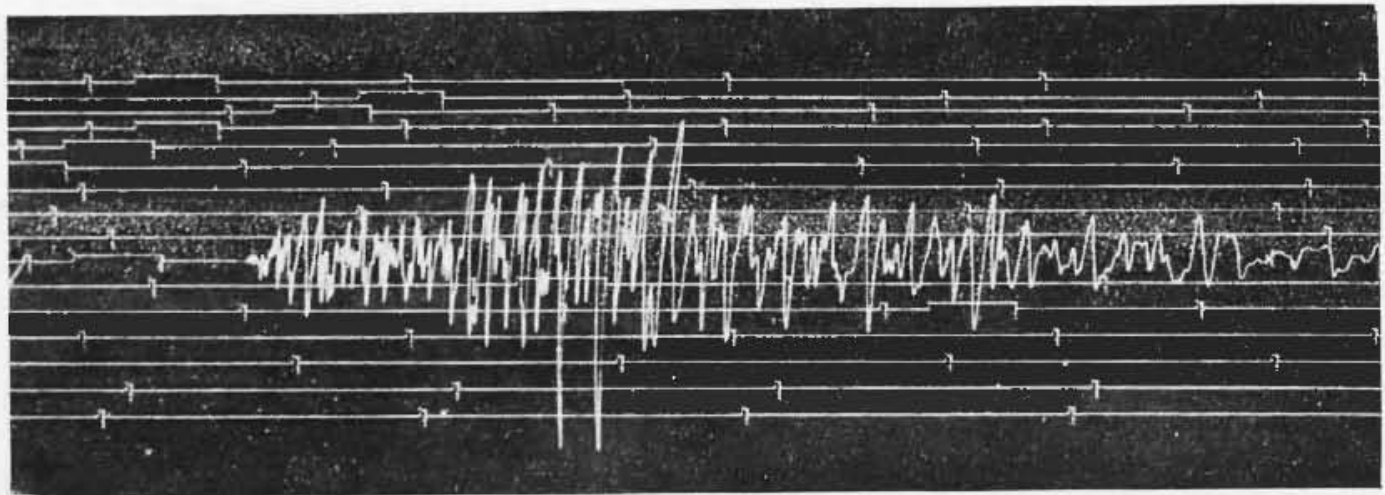

Firr. 5-Scossa X. 5 del $15-1-1968$ alle $03^{\text {no }} 00^{\mathrm{m}}$, (Wiechert $80 \mathrm{~kg}$, componente Z).

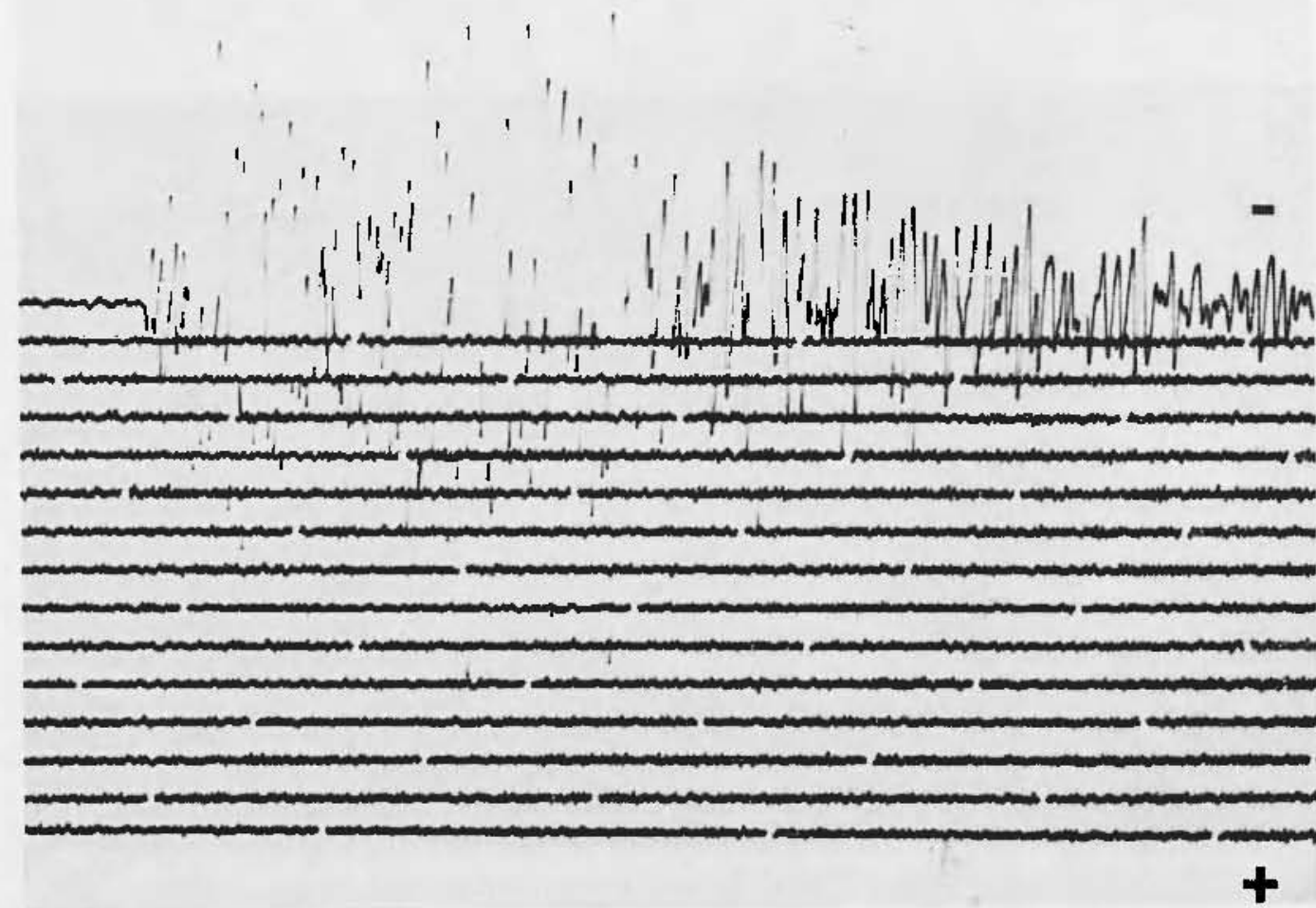

Fig. 6 - Seossa N. I4 (Girlanda, componente Z). 


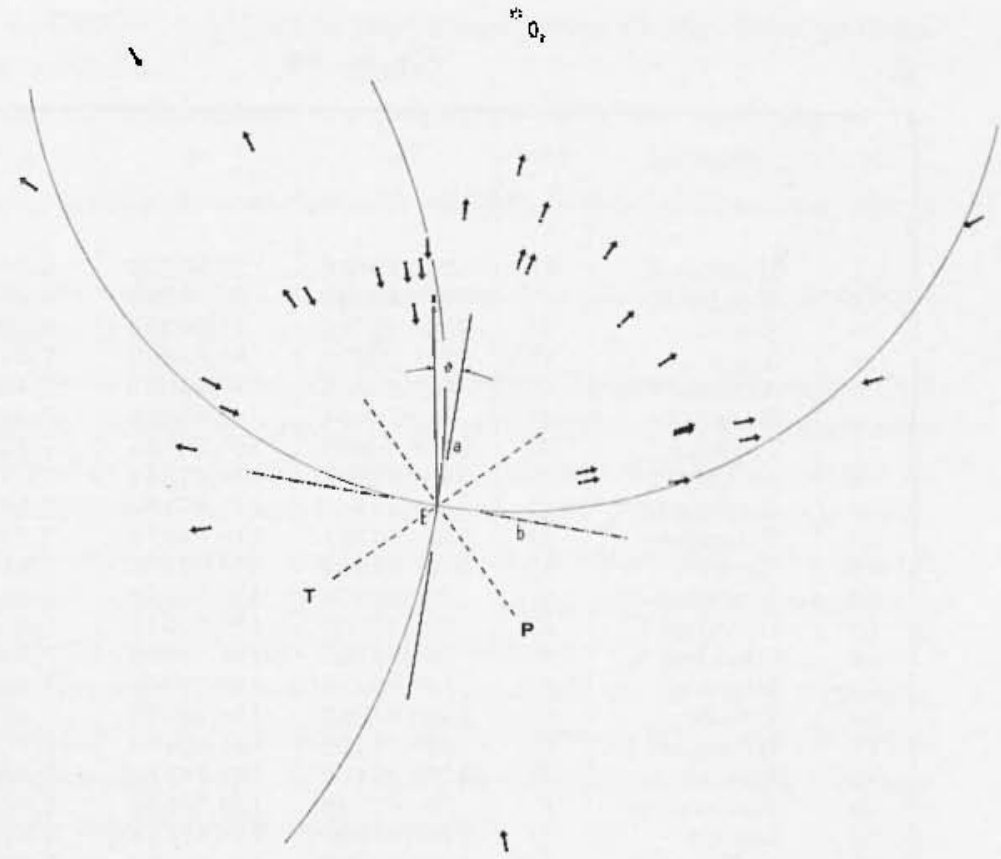

Fig. 7

inizio di stazioni lontane che, al contrario, non sono state prese in considerazione da Marcelli e Pannocebia (12).

Infine, nell'intento e ri completare questa ricerca e di poter proIurre qualche altro elemento a sostegno della superficialiti diei terremoti deI Belice, si sono consirlerati i versi rlei primi impulsi della scossa principale (X. $5 ; M=5,9$ ) in oltre trenta stazioni.

I rati racolti, unitamente agli elementi calcolati per rappresentare in proiezione stereografica Ia distribuzione delle dilatazioni e delle compressioni, sono riportati in 'Tab. IV. La costruzione grafiea conseguente ì quella di Fig. $\bar{\tau}$, ove a e $b$ sono le tangenti alle circonferenze di centro $\mathrm{O}_{1}$ e $\mathrm{O}_{2}$ rispettivamente, intersecantisi ortogonalmente nell'epicentro $\mathbf{E}=\mathbf{I}$, e delimitanti le due coppie di zone a dilatazione e compressions.

Complessivamente l'individuazione grafica rlel piano principale e diel piano ausiliario, aventi nel piano di superficie le trace a e b ris]etivamente, deve ritenersi soddisfacente. Infatti, la relativa abbondanza di dati nel I e nel IV quadrante, nonchi la favorevole posi- 
Tahยมก. I

\begin{tabular}{|c|c|c|c|c|c|c|}
\hline N. & Stazioni & $1 / \mathrm{C}$ & $a$ & $\Delta$ & $v$ & $\delta$ \\
\hline 1 & Messina 1: & $\mathrm{C}$ & $78^{\circ}, 42066$ & 20,00868 & 6,302 & 0,559 \\
\hline 2 & Reggio $(\mathrm{C}$. & c: & $81^{\circ}, 46222$ & 20,06396 & 6,313 & 0,563 \\
\hline 3 & Roma & J) & $354^{\circ}, 31717$ & $4^{0}, 09322$ & (6,984 & 0,783 \\
\hline 4 & Alger & $\mathrm{C}$ & $274^{\circ}, 25278$ & $8^{\circ}, 05086$ & 7,399 & 0,900 \\
\hline 5 & Athenai & C & $85^{\circ}, 75555$ & $8^{0}, 43037$ & $7,+21$ & 0,906 \\
\hline i & Beograt & J) & $36^{\circ}, 18604$ & $8^{0}, 93064$ & 7,447 & 0,913 \\
\hline 7 & Tortosia & I) & $205^{\circ}, 60667$ & $10^{\circ}, \overline{0} 4755$ & 7,536 & 0,936 \\
\hline 8 & Stuttgart & J) & $346^{\circ}, 82861$ & $11^{0}, 27850$ & 7,572 & 0,946 \\
\hline 9 & Karlsiuhe & J) & $356^{\circ}, 04445$ & $11^{\circ}, 67490$ & 7,593 & 0,952 \\
\hline 10 & Erlangen & 1) & $353^{\circ}, 54181$ & $11^{0}, 85374$ & 7,599 & 0,953 \\
\hline II & fiarcy & $\mathrm{C}$ & $325^{\circ}, 34944$ & $11^{0}, 97710$ & 7,610 & 0,950 \\
\hline 12 & Nielziea & C: & $22^{\circ}, 02378$ & $12^{\circ}, 72270$ & 7,641 & 0,964 \\
\hline 13 & Jstambul U. & C & 700,43500 & $12^{\circ}, 72747$ & 7,646 & 0,060 \\
\hline 14 & Istanbul K. & C & 700,38130 & $12^{\circ}, 78645$ & $7,6+0$ & 0,067 \\
\hline 15 & Kranow & $\mathrm{C}$ & 190,80278 & $13^{\circ}, 17968$ & 7,664 & 0,970 \\
\hline 16 & Toledo & (C) & $284^{\circ}, 04083$ & $13^{\circ}, 49072$ & 7,684 & 0,975 \\
\hline 17 & Kichiney & (! & $46^{\circ}, 74422$ & $14^{\circ}, 83176$ & 7,744 & 0,901 \\
\hline 18 & Kew & 1) & $329^{\circ}, 32175$ & $16^{\circ}, 46518$ & 7.820 & 1,011 \\
\hline 19 & Simferopol & c & $59^{\circ}, 07722$ & $17^{\circ}, 30532$ & 7,863 & 1,021 \\
\hline 20 & lishoa & 1) & 2790,77500 & $17^{\circ}, 45771$ & 7,871 & 1,023 \\
\hline 21 & Kobenalisn & 1) & 3580,85092 & $77^{\circ}, 86870$ & 7,806 & 1,030 \\
\hline 22 & Porto & I) & 2990,32806 & $18^{\circ}, 64865$ & 7,057 & 1,045 \\
\hline 23 & Uppsala & C & 60,09861 & $22^{\circ}, 24365$ & 8,240 & 1,115 \\
\hline 24 & Moska & c: & $35^{\circ}, 21805$ & $24^{\circ}, 06487$ & 8,415 & 1,158 \\
\hline 25 & Poulliovo & $c$ & $21^{0}, 16264$ & $24^{\circ}, 58370$ & 8,462 & $1,1,99$ \\
\hline 26 & Goris & C & $75^{\circ}, 90083$ & $25^{\circ}, 95896$ & 8,589 & 1.199 \\
\hline 27 & Tabriz & C & 790,12306 & $26^{\circ}, 16312$ & 8,608 & 1,204 \\
\hline 28 & Apatity & C & $14^{\circ}, 59750$ & $31^{\circ}, 88124$ & 9,105 & 1,319 \\
\hline 29 & Bangui & I) & $1700^{\circ}, 05674$ & $33^{\circ}, 68113$ & 9,246 & 1,351 \\
\hline 30 & Godhavn & $c$ & $333^{\circ}, 41061$ & $46^{\circ}, 94321$ & 10,148 & 1,551 \\
\hline 31 & Shillong & 1) & $75^{0}, 26861$ & $66^{\circ}, 37846$ & 11,297 & 1,704 \\
\hline 32 & Tulsa & C & $309^{\circ}, 01953$ & $81^{\circ}, 43581$ & 12,226 & 1.985 \\
\hline 33 & Mincral & D) & $327^{\circ}, 04999$ & $01^{\circ}, 81192$ & 12,897 & 2,121 \\
\hline
\end{tabular}

I) = Dilatazione; $\quad \mathrm{C}=$ (ompressione; $\alpha=$ Azimut rispetto all'epicentro; $\Delta=$ Distanza epicentrale; $v=$ Velocitis apparente in $\mathrm{km} . \mathrm{sec}^{-1} ; \delta \equiv$ Proiczione stereografiea del raggio sisunico rettificato.

zione di qualehe stazione nel II, restringono le incertezze sugli azimuts a. \pm 20 .

Egualmente buona l'omogeneità relativa dei versi dei primi impulsi, constatato che i soli dati di Garey e Godhavn, entrambe nel IV qualdante, sono in disaccordo ron la disposizione delle zone a dilatazione e compressione (Fig. T). 
I dati di 'lab. IV e quindi la rappresentazione di Fig. 7 ammettono la seguente soluzione:

- Piano di dislocazione con azimut $\mathrm{X} 11^{\circ} \mathrm{E}$ ed inclinazione $55^{\circ}$ ¿ $\mathrm{N} 790 \mathrm{~W}$.

- Piano ausiliario con azimut $\mathrm{N} 101^{\circ} \mathrm{E}$ ed inclinazione $22^{\circ}$ a N $11^{\circ} \mathrm{j}$.

- Azimuts delle direzioni di massima $(P)$ e minima $(T)$ pressione rispettivamente $\mathrm{N} 34^{\circ} \mathrm{W}$ e $\mathrm{N} 56^{\circ} \mathrm{H}$.

Preso atto che un piano di dislochzione disposto come sopra non trova riscontro preciso e sicuro nella cartografia e nella letteratura geologica rlell'area interessata, ò peró da rilevare che per la scossa in questione la direzione di massinu pressione $(P)$ ì sensibilmente ortogonale all'asse della dorsale Castelvetrano-M.te Finestrelle $\left(\mathbf{I}_{1}\right\rangle$ e quindi all'allineamento epicentrale determinato. Quest'ultimo ba pertanto lo stesso azimut del piano delle massime tensioni.

Quanto rilevato, a nostro avviso, costituisce un ulteriore elemento a favore della presunta correlazione fra l'attivitì sismien manifestatasi e Ia neotettonica della Valle del Belice, ed indirettamente, un ulteriore sostegno della superficialitia degli ipocentri.

\section{BIBLIOG R $\Lambda F I \Lambda$}

(1) Benso E., 1961. - Studi ed indagini per rieerehe di idrocarburi. Regione Siciliana.

(2) Bosi C., Cavallo R., Manfredini M., 1968. - Il terremolo della Valle del Beliee del Gennuio 1968. "Consigrlio Nazionale delle Ricerche, Centro di studio per la Geologia Teeniea ", Febloraio.

(3) Caflisch L., Scimidit di Frimnero P., 1967. - Jievoluzione paleografica della Sicilia e sue relazioni con la lettonica $e$ ln unflogenesi. "Mrem. Soc. Geol. Ital, ", VI, 4, 449-475.

$\left({ }^{4}\right)$ CaLor P., Peronaci F, 1948. - Il terromoto del Turkestan del 2 Novembre 1946, "Annali di Geofisica ", I, 2, 246-252.

$\left.{ }^{5}\right)$ De Panfilis M., Marcelli L., 1968. - Il periodo sismieo della Sieilia Occidentale iniziato il 14-1-1968. "Annali di Geofisican, XXI, 4, 343422 .

(6) Di Filippo D., Peronaci F., 1962. - Terromoti di frattura e relazioni con la tettonica nelle Alpi orientali. "Annali di Geofisiea ", XV, 2-3, 195-224.

(7) Evison F. F., 1970. - Seismogenesis, "Tectonophysics", IX, 2-3. 
(") GuRlanda A., 1968. - Liallivita sismica della Sirilin Occidentale dal 11 Gennnio 1968. "Enc. della Scienza e della Teeniea ", Momlathri, Milano.

(9) Gintanida A., 1970. Comunieazione personale su ricerehe non pubblicate.

(10) Giross ]), 1939. Creep of roeks. "Journal of (feology ", XLVII, 225.

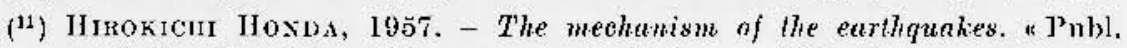
of the Dominion Observatory of Ottawa", XX, 2.

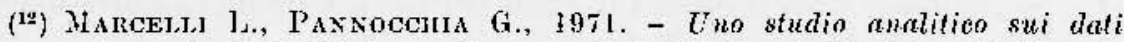
ipocentrali di 10 torremoti acemuti in Sicilia Occidentale nel fennaio 1968. "Annali di Geofisica", XXIV, 2, 287-306.

(13) Mrcuessos J., 191 7 . . Elastic viscous fiow (J). "Journal of Geology ", $\mathrm{XXV}$.

(1) Nezhi Canifez, S. Batamir licen, 1967, - Computer determinations for the fauli-plane solutions in and near Anatolin. "Tectonophysies", IV, $3,235-244$.

(15) Rigo de Rigia F., 1956. - Olistostromi neogenici in Sicilia. "Boll, Soe. Geol, 1tal. ", LXXV, 3, 185-215.

(5) RiTsEm A 1. R, 1967. - Mechanisms of european earthquakes. "Tectonophysics", IV, 3, 247-259.

(17) Varde P. li., 1969. - Tentativo di controllo del periodo sismico siciliano inizinto il 14-1-1968. "Annali di Geofisica " XXII, 1, 57-84. 